\title{
Identifying synthetic lectins from a competitive screen for the detection of prostate câncer.
}

\author{
Mary Grace Hollenbeck, Anna A. Blevins, Erin E. Gatrone, Tanya Hundal, Kathleen O’Connell and John J. \\ Lavigne*
}

Department of Chemistry \& Biochemistry, University of South Carolina, 631 Sumter Street, Columbia, SC, USA 29208. Tel: (803)777-2295; Fax: (803)-777-9521; E-mail: Lavigne@ sc.edu

KEYWORDS. Synthetic Lectin, boronic acid, phenotypic screen, competitive screen, prostate cancer

\section{Experimental}

General Methods. All chemicals were purchased from Millipore Sigma or Fisher Scientific and used without further purification. TentaGel resin (Cat. No. MB-300-002; loading level 0.25-0.3 mmol/g) was purchased from Rapp Polymere. Cell growth media and fetal bovine serum was purchased from VWR International, LLC (West Chester, PA). BCA protein quantification kits were purchased from Thermo Scientific. The SL hits were sent to Tufts University Core Facility (Medford, MA) for sequencing via Edman Degradation.

Synthesis of SL Library. Standard Fmoc / HBTU chemistry was used to attach a peptide backbone to the TentaGel resin. TentaGel was chosen because it is dynamic in aqueous and organic solvents, stable in acidic conditions, has a high loading capacity, which is important for peptide sequencing, and contains a large number of beads per gram, which is important for sequence diversity. The peptides in this library have the general sequence Ac-R-X-D*-X-X-X-D*-X-B-B-R-M-resin where B denotes beta-alanine, $\mathrm{D}^{*}$ denotes diamino butanoic acid functionalized with 4 -formylphenyl boronic acid, and $\mathrm{X}$ denotes a random amino acid. Cysteine, methionine, proline, histidine, glutamic acid, aspartic acid, lysine, tryptophan and isoleucine were excluded as potential random amino acid, X, in the peptides sequence. Cysteine and methionine were excluded to avoid issues during cleavage because these amino acids contain thiol moieties which are integral in how the peptide is cleaved from the resin. Proline was excluded so that the peptides would not have any turns. Histidine was avoided to allow for more straightforward analysis of side-chain charge. Glutamic and aspartic acids were excluded because they have negatively charged side chains. Lysine was not used in order to simplify side-chain deprotection strategies. Tryptophan was excluded because it has a big and bulky side chain that is redox active. Isoleucine has the same molecular weight as leucine and was avoided to simplify sequencing. $250 \mathrm{mg}$ of resin beads were weighed out and soaked in DMF for 10 minutes. 8 equivalents of methionine and HBTU were dissolved in $10 \mathrm{~mL}$ of $5 \% \mathrm{~N}$-methylmorpholine / DMF. The solution was left at room temperature for 10 minutes before mixing with the resin. After tumbling for 1 hour, the beads were washed with DMF and methanol. A ninhydrin tests were performed to determine if the amino acids had coupled. The Fmoc group was removed by tumbling the beads in $10 \mathrm{~mL} \mathrm{20 \%}$ piperdine in DMF for 30 minutes. This process was repeated with arginine and then two $\beta$-alanines. The random amino acid, $\mathrm{X}$, was coupled to the beads using the split-and-pool method. Briefly, the beads were split into 12 different reaction vessels that each contain a different activated amino acid. After coupling, the beads were combined back into one vessel and deprotected using $20 \%$ piperdine in DMF. Since the next position is fixed with Dab, the beads are coupled to the amino acid and deprotected in the same vessel. The next 6 amino acids are coupled depending on the fixed or random position of that particular amino acid. Once all of the amino acids are coupled, the end of the peptide strand is acylated by tumbling the resin in $10 \mathrm{~mL}$ of $90 \%$ dichloromethane, $5 \%$ acetic anhydride, and 5\% pyridine. The ivDde protecting group of Dab was removed by using $10 \mathrm{~mL}$ of a $5 \%$ hydrazine / DMF solution.

Incorporation of Phenyl Boronic Acid. 2-Formylphenyl boronic acid (8eq) was dissolved in a mixture of $10 \%$ methanol in DMF. The resin beads, along with activated $3 \AA \AA$ molecular sieves, were added into the solution and tumbled at $37^{\circ} \mathrm{C}$ overnight. $\mathrm{NaBH}_{4}(8 \mathrm{eq}) \mathrm{was}$ added before and the reaction was left uncapped to allow $\mathrm{H}_{2}$ gas to escape for 45 -minutes. The resin was left to tumble at $37^{\circ} \mathrm{C}$ for $4-$ hours. The resin was then separated from the molecular sieves, based on density, using dichloromethane. This was followed by washing the beads with copious amounts of DMF and methanol.

Removal of Acid Laible Groups. Molecular sieves were removed, and the beads were transferred to a Flacon tube. After the boronic acid moiety was coupled, $10 \mathrm{~mL}$ of $95 \%$ trifluoroacetic acid (TFA), $2.5 \%$ water, and $2.5 \%$ triisopropylsilane $(9.5 \mathrm{ml}, 250 \mu \mathrm{L}, 250 \mu \mathrm{L}$ resp.) was added and the resin was tumbled for 1 hour to deprotect the acid-labile protecting groups. The TFA wash was repeated one more time. The resin was then washed with DMF and methanol. SLs were tumbled with PBS without glycerol (pH 7.25) twice for 10 minutes before future use.

Library Screen Using Single Analyte Method. $2 \mathrm{mg}$ of library resin was rinsed in phosphate buffer saline (PBS) twice and then preincubated with $1 \%$ BSA in PBS with $10 \%$ glycerol for 15 minutes. This pre-incubation was to reduce the nonspecific binding between the SL and proteins. The 1\% BSA in PBS with $10 \%$ glycerol solution was then removed from the beads and $1 \mathrm{~mL}$ of a $0.0002 \mathrm{mg} / \mathrm{mL}$ solution of the fluorescently labeled prostate membrane extract in PBS was incubated with the beads for 12 hours at room temperature with gentle shaking. The extract was then removed, and the beads were washed three times by adding $1 \mathrm{~mL}$ of PBS. Beads were then viewed on a fluorescent microscope. 
Library Screen Using Dual Dye Method. The same protocol outline for the single analyte was followed, however, instead of using 1 $\mathrm{mL}$ containing $0.0002 \mathrm{mg}$ of both analytes to give a total concentration of $0.0004 \mathrm{mg} / \mathrm{mL}$ of protein, only $0.0001 \mathrm{mg}$ was use of each to make a total concentration of $0.0002 \mathrm{mg} / \mathrm{mL}$.

Microscopy. All fluorescent images were taken using a Leica MZ 16F microscope equipped with a GFP3 filter (excitation 450-490 nm; emission filter 500-550 nm), DSR filter (excitation 510-560 nm; emission filter 590-650 nm), and a QImaging MicroPublisher 5.0 RTV digital camera. Images were analyzed using a MATLAB algorithum which provided fluorescent intensities.

Resynthesis of SL Hits. $500 \mathrm{mg}$ of resin was swelled in $10 \mathrm{~mL}$ of DMF for 10 minutes before being put onto a Protein Technologies Peptide Synthesizer (PS3). The beads were removed from the PS3 after all 12 amino acids has been added. Once all of the amino acids were couples, the ivDE protecting group on Dab was removed by washing the beads three times in 5\% hydrazine / DMF for 1 hour at room temperature. Afterwards, the phenyl boronic acid was added and the acid laible groups were removed using the protocols outlined above.

MS/MS Analysis. To verify that the PS3 coupled the correct sequence onto the resin MS/MS analysis was used. The peptide was then cleaved from the resin, the beads were treated with $50 \mu \mathrm{l}$ of $40 \mathrm{mg} / \mathrm{ml}$ cyanogen bromide $(\mathrm{CNBr})$ in $0.1 \mathrm{M} \mathrm{HCl}$ for 16 hours in the dark. The beads were removed from the solution and the excess CNBR, TFA, and water were removed under reduced pressure by using an AES1010 SpeedVac system. The cleaved peptides were re-dissolved in $10 \mu 1$ of $0.1 \%$ TFA in water. A solution of 50:50:0.1 acetonitrile/water/TFA was saturated with $\alpha$-cyano-4-hydroxycinniamic acid (CHCA). On a MALDI plate, $1 \mu$ of this matrix containing solution was mixed with $2 \mu \mathrm{l}$ of the cleaved SL solution and dried under ambient conditions. Samples were then analyzed by MALDITOF/MS and MS/MS techniques.

Binding Studies with Fluorescent-labeled Membrane Extracts. Approximately $2 \mathrm{mg}$ of SL was rinsed in PBS twice and then preincubated with $1 \%$ BSA in PBS with $10 \%$ glycerol for 15 minutes. This pre-incubation was to reduce the nonspecific binding between the SL and proteins. The $1 \%$ BSA in PBS with $10 \%$ glycerol solution was then removed from the beads and $1 \mathrm{~mL}$ of a $0.0002 \mathrm{mg} / \mathrm{mL}$ solution 45 of the fluorescently labeled prostate membrane extract in PBS was incubated with the beads for 6 hours at room temperature with gentle shaking. After incubation, the beads were rinsed with PBS three times, imaged with a fluorescent microscope, Leica MZ 16F, and analyzed by Matlab R2012a. The output was analyzed by linear discriminant analysis using SYSTAT 11.00.01.

Cell Culture. VWR supplied all of the supplements and media needed for cell culture. RWPE-1, WPE1- NA22 and WPE1-NB14 cells were grown in Keratinocyte Serum Free Media (K-SFM) supplemented with human recombinant epidermal growth factor (rhEGF) and bovine pituitary extract (BPR). PC3, LNCaP, HCT 116 and LoVo cells were grown in RPMI 1640 media with 10\% feline bovine serum (FBS). DU145 CCD-841-CON and HT29 cells were grown in Eagle's Minimum Essential Medium Cells supplemented with 10\% v/v of FBS and 1\% antibiotics / anti-mycotic were passaged upon $75 \%$ confluence in T75 Flasks.

Fluorescent Labeling of Cell Membrane Extracts. The Qiagen Plasma Membrane Protein Kit was used to extract plasma membrane proteins and glycoproteins. This kit isolates all membrane bound proteins not only cancer associated markers. The extract was spun into carbonate buffer $\mathrm{pH} 9.8$ and a BCA assay was performed to obtain the concentration of the extract. The amount of protein needed for library screening was added to carbonate buffer to a total volume of $600 \mu \mathrm{L}$. Depending on which dye was being used to label, 0.07 $\mu$ mol FITC or NHS-rodamine was added and the solution was incubated at $37^{\circ} \mathrm{C}$ and tumbled for $1 \mathrm{hr}$. Amicon Pro Centrifugal Filter $(10 \mathrm{kDa})$ were used to remove excess dye and to concentrate the proteins. 


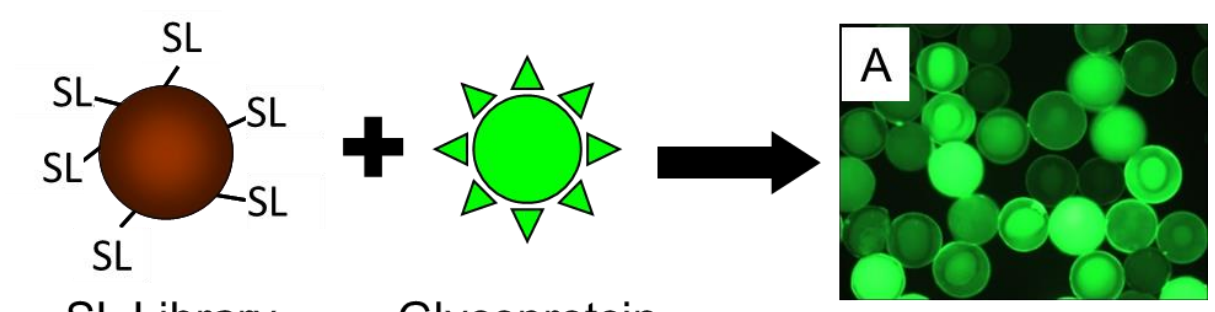

\section{SL Library Glycoprotein}

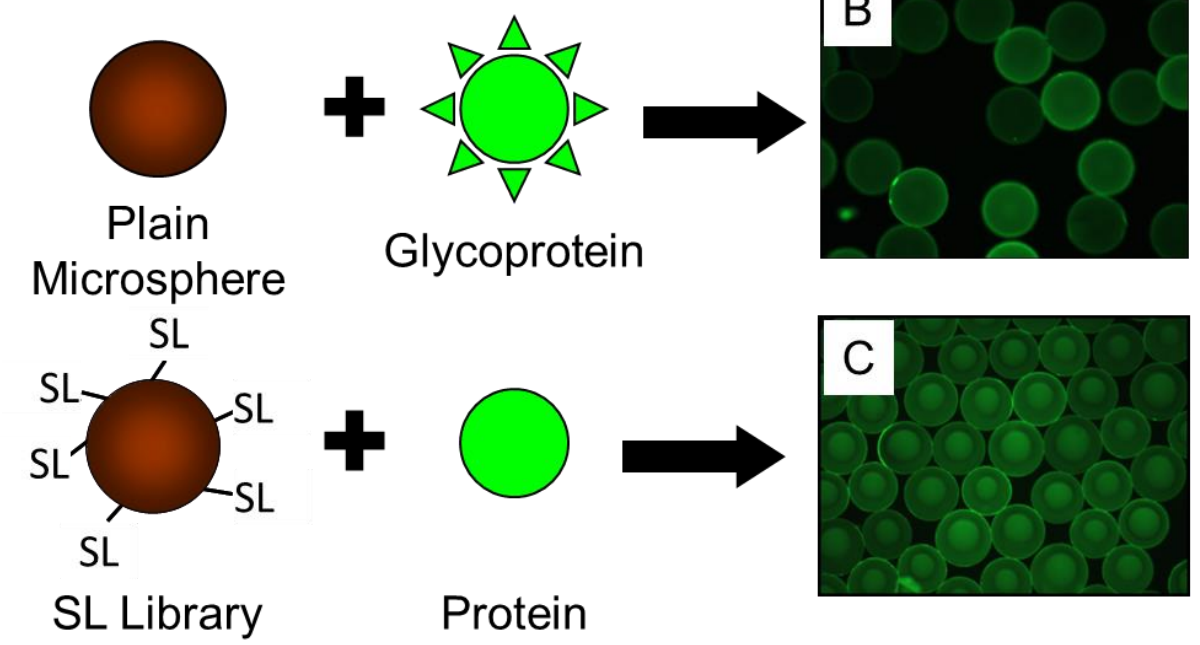

Figure S1. Glycan binding experiments showing binding between (A) the SL library and the fluorescein tagged glycoprotein, (B) Tentagel resin binding fluorescein tagged glycoprotein, and (C) the SL library and the fluorescein tagged protein. Since A has the greatest fluorescent intensity, these results support the idea that the peptide of the SL library binds the glycan portion of the protein. 
Table S1. Leave-one-out cross validation classification matrix for the 5 SL-array based assay.

\begin{tabular}{|l|c|c|c|c|c|c|c|}
\hline & DU145 & LNCaP & PC3 & RWPE-1 & WPE1-NA22 & WPE1-NB14 & \% Correct \\
\hline DU145 & 16 & 1 & 0 & 2 & 1 & 0 & 80 \\
\hline LNCaP & 0 & 12 & 6 & 0 & 0 & 1 & 63 \\
\hline PC3 & 0 & 7 & 11 & 0 & 0 & 0 & 61 \\
\hline RWPE-1 & 0 & 0 & 0 & 18 & 1 & 0 & 95 \\
\hline WPE1-NA22 & 0 & 0 & 0 & 0 & 20 & 0 & 100 \\
\hline WPE1-NB14 & 0 & 1 & 0 & 0 & 0 & 19 & 95 \\
\hline
\end{tabular}

Edman Degradation: SL hits were sent off to Tufts University Core Facility for sequencing. The following spectra were outputted. All of the peptides have the same general sequence, R-X-D*-X-X-X-D*-X-BBRM, where X represents a random amino acid, $D^{*}$ represent diaminobutyric acid, and $\mathrm{B}$ represent $\beta-\mathrm{Ala}$. MRBB is a conserved sequence so the spectra are not shown. The N-terminus will be labeled as amino acid 1.

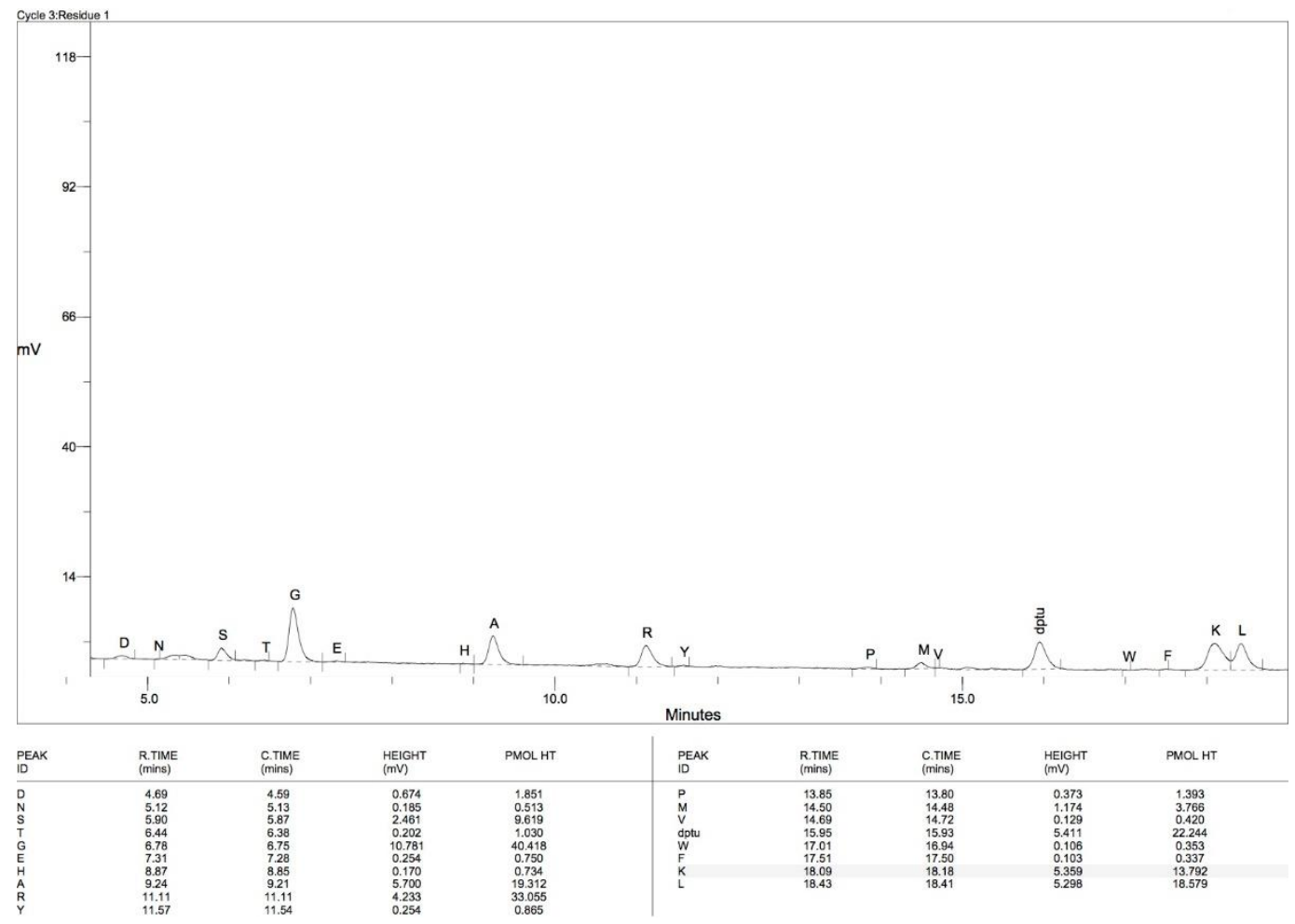

Figure S1: Edman Sequence Results for SL10 Amino Acid 1

The sequence of SL10 is R-L-D*-A-R-S-D*-G-B-B-R-M. R is a fixed position in the SL library. 


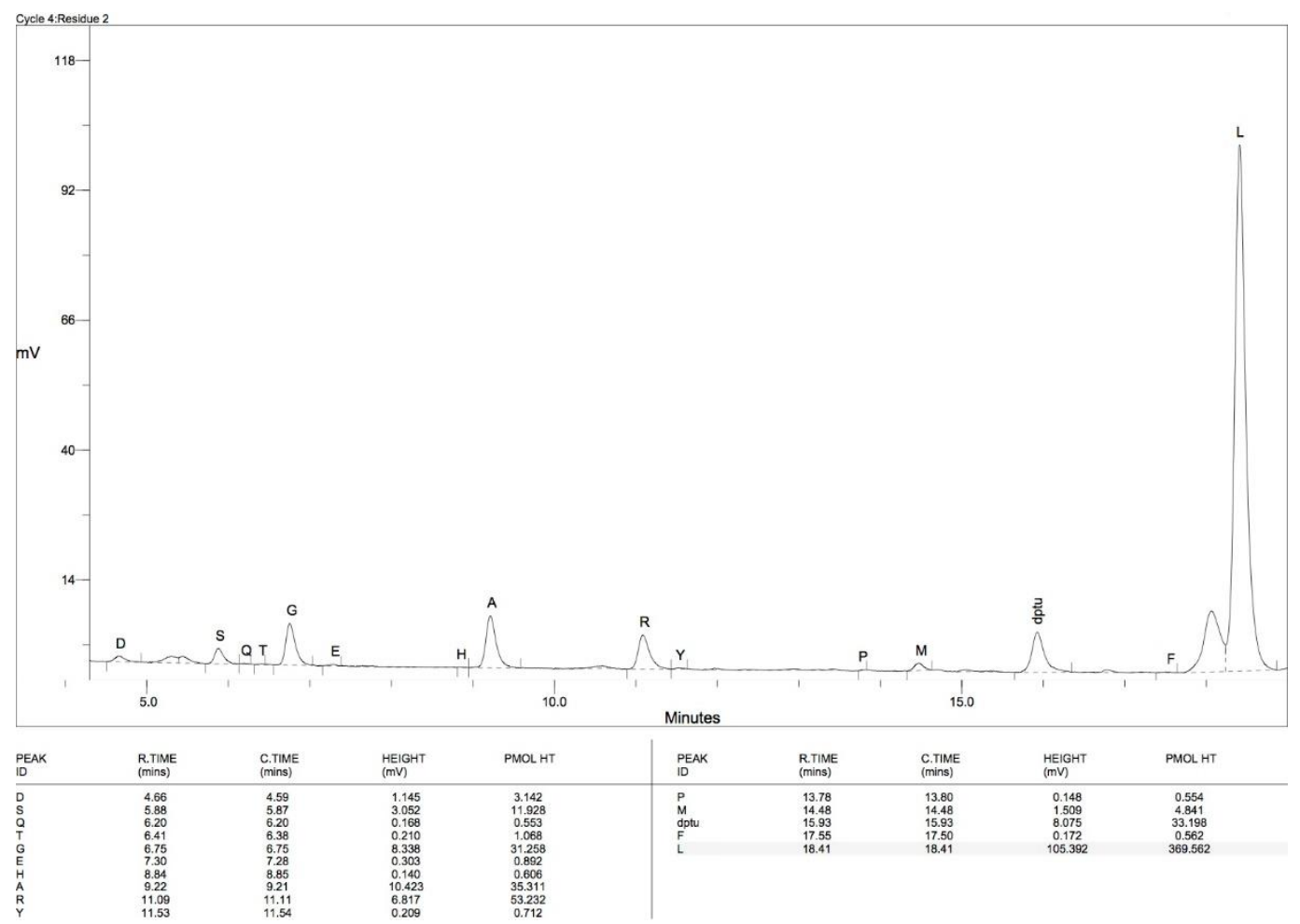

Figure S2: Edman Sequence Results for SL10 Amino Acid 2

The sequence of SL10 is R-L-D*-A-R-S-D*-G-B-B-R-M. Leucine was present in the first randomized position.

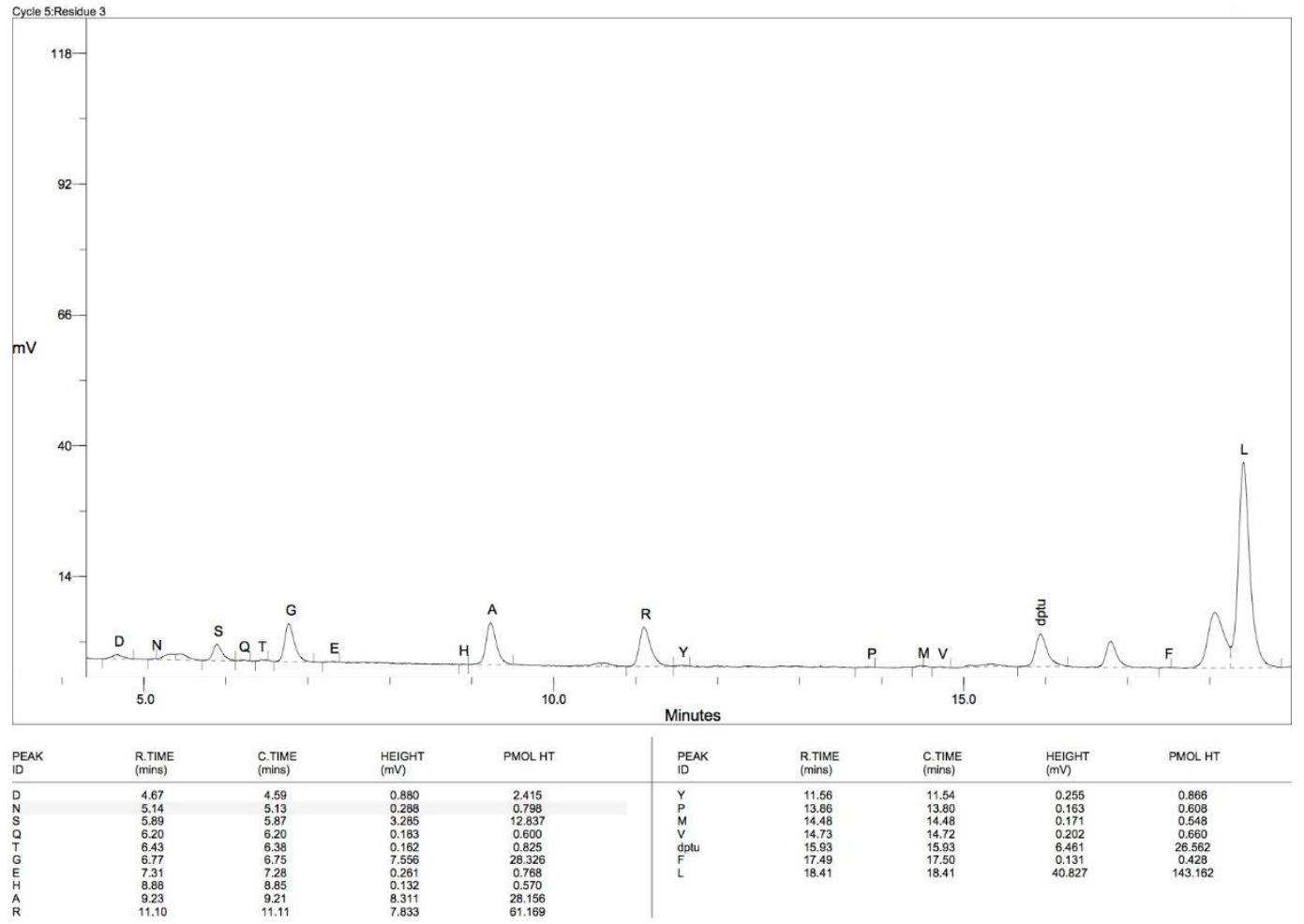

Figure S3: Edman Sequence Results for SL10 Amino Acid 3

The sequence of SL10 is R-L-D*-A-R-S-D*-G-B-B-R-M. D* is a fixed position in the library and is not recognized by Edman Degradation. The fixed position of this AA identifies where it falls in the SL sequence. It is also important to note that while $\mathrm{L}$ does show in the cycle of the sequence, the amount decreases from the cycle before. 


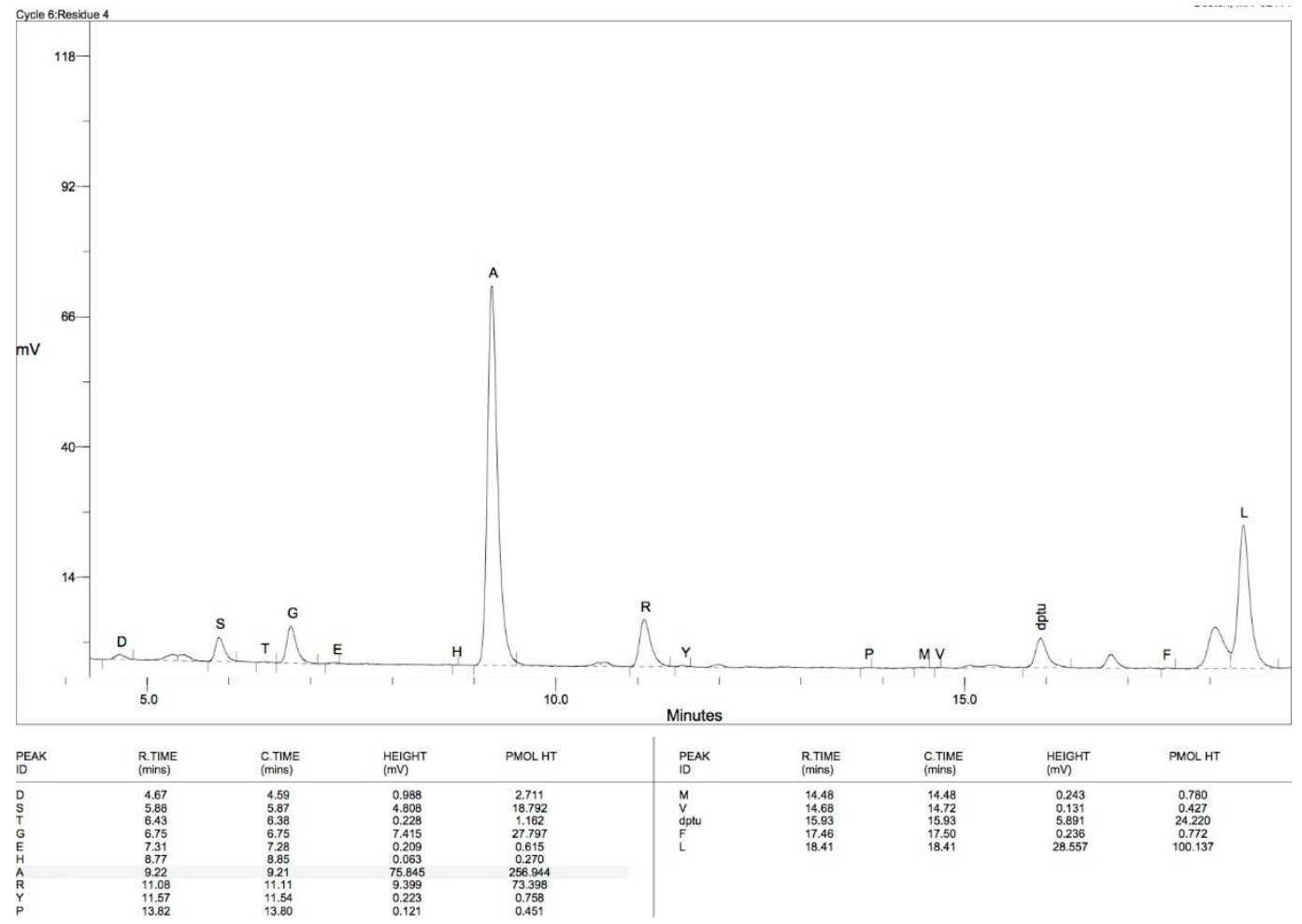

Figure S4: Edman Sequence Results for SL10 Amino Acid 4

The sequence of SL10 is R-L-D*-A-R-S-D*-G-B-B-R-M. Alanine was present in the second randomized position.

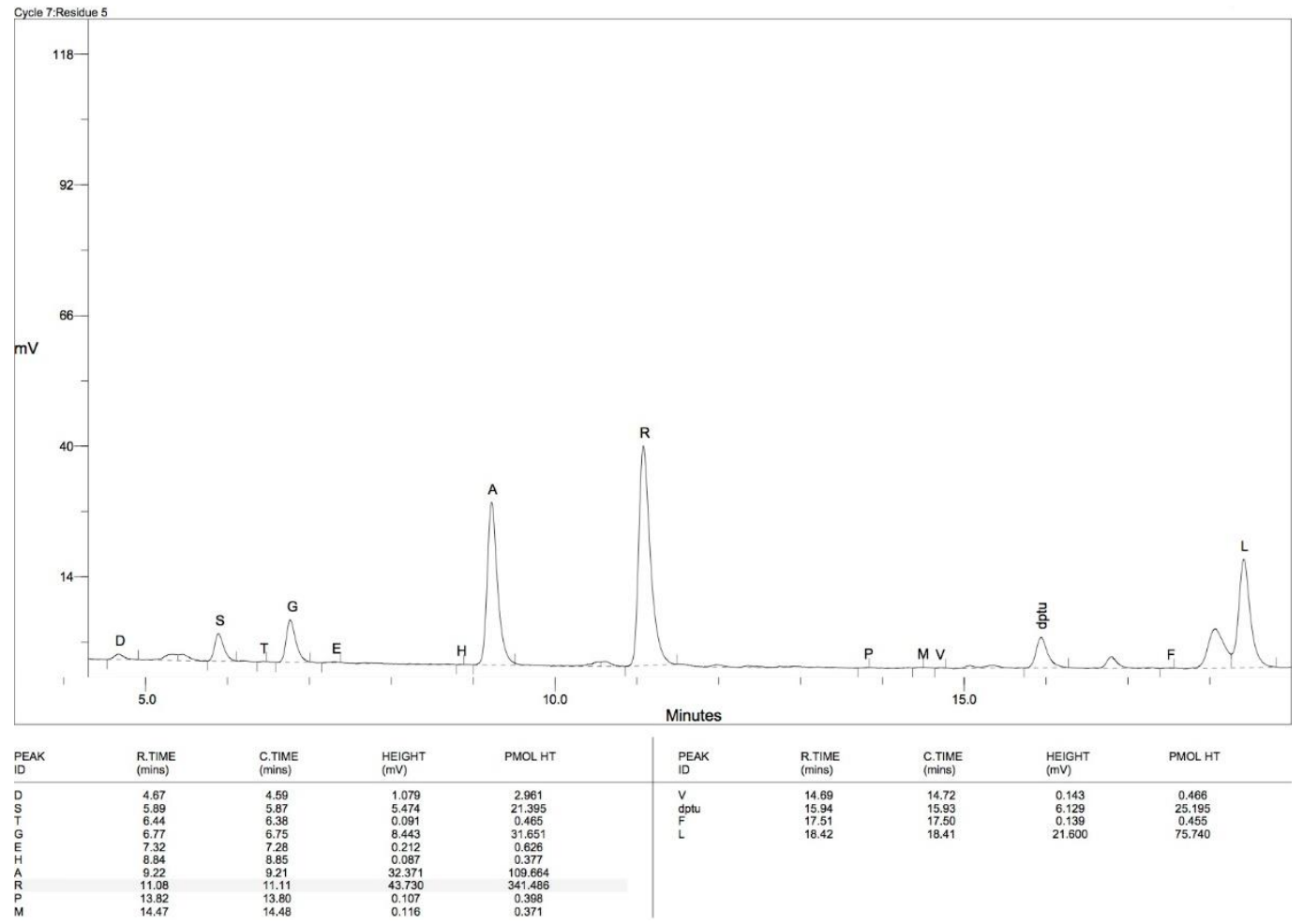

Figure S5: Edman Sequence Results for SL10 Amino Acid 5

The sequence of SL10 is R-L-D*-A-R-S-D*-G-B-B-R-M. Arginine was present in the third randomized position. 


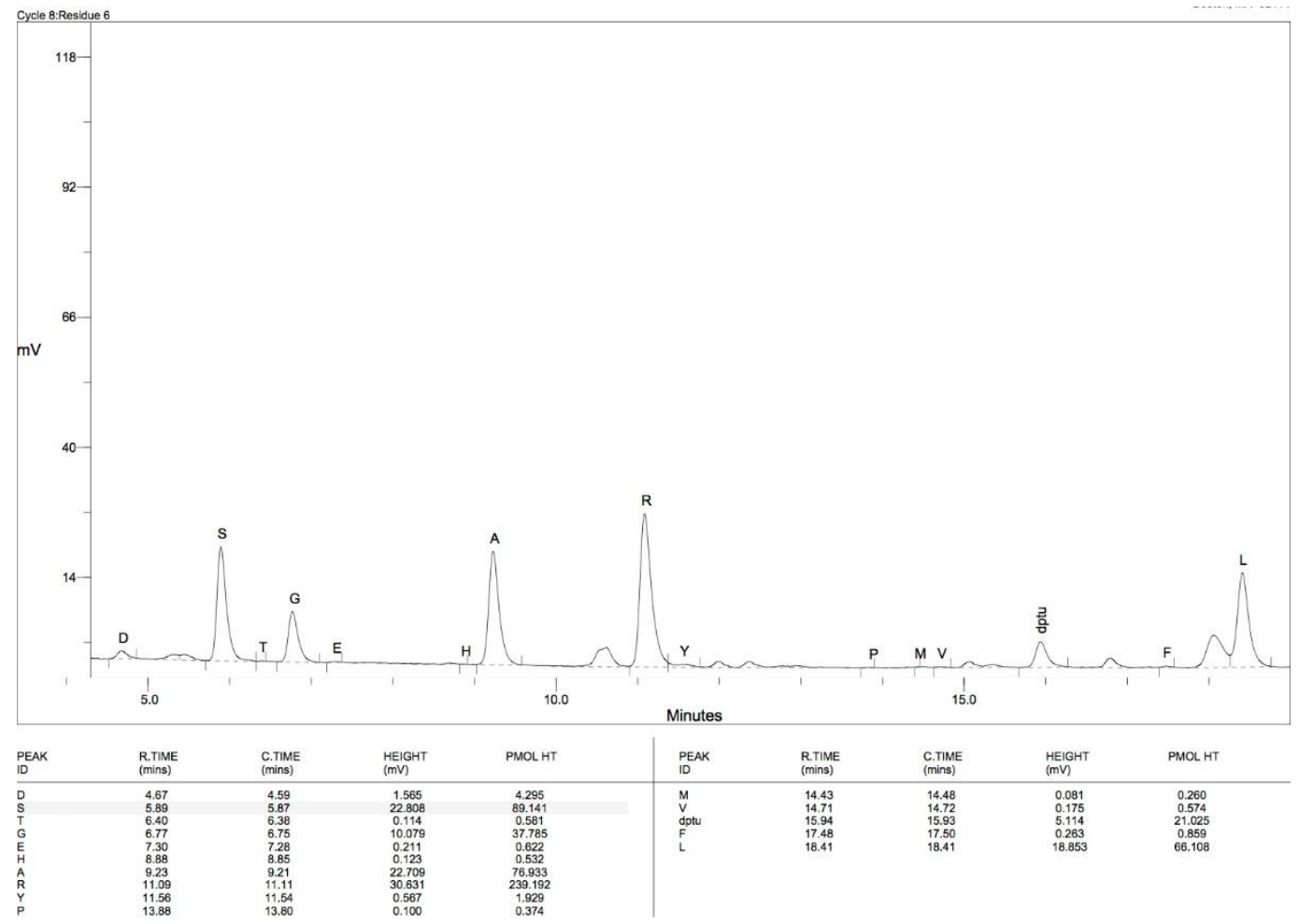

Figure S6: Edman Sequence Results for SL10 Amino Acid 6

The sequence of SL10 is R-L-D*-A-R-S-D*-G-B-B-R-M. Serine was present in the fourth randomized position.

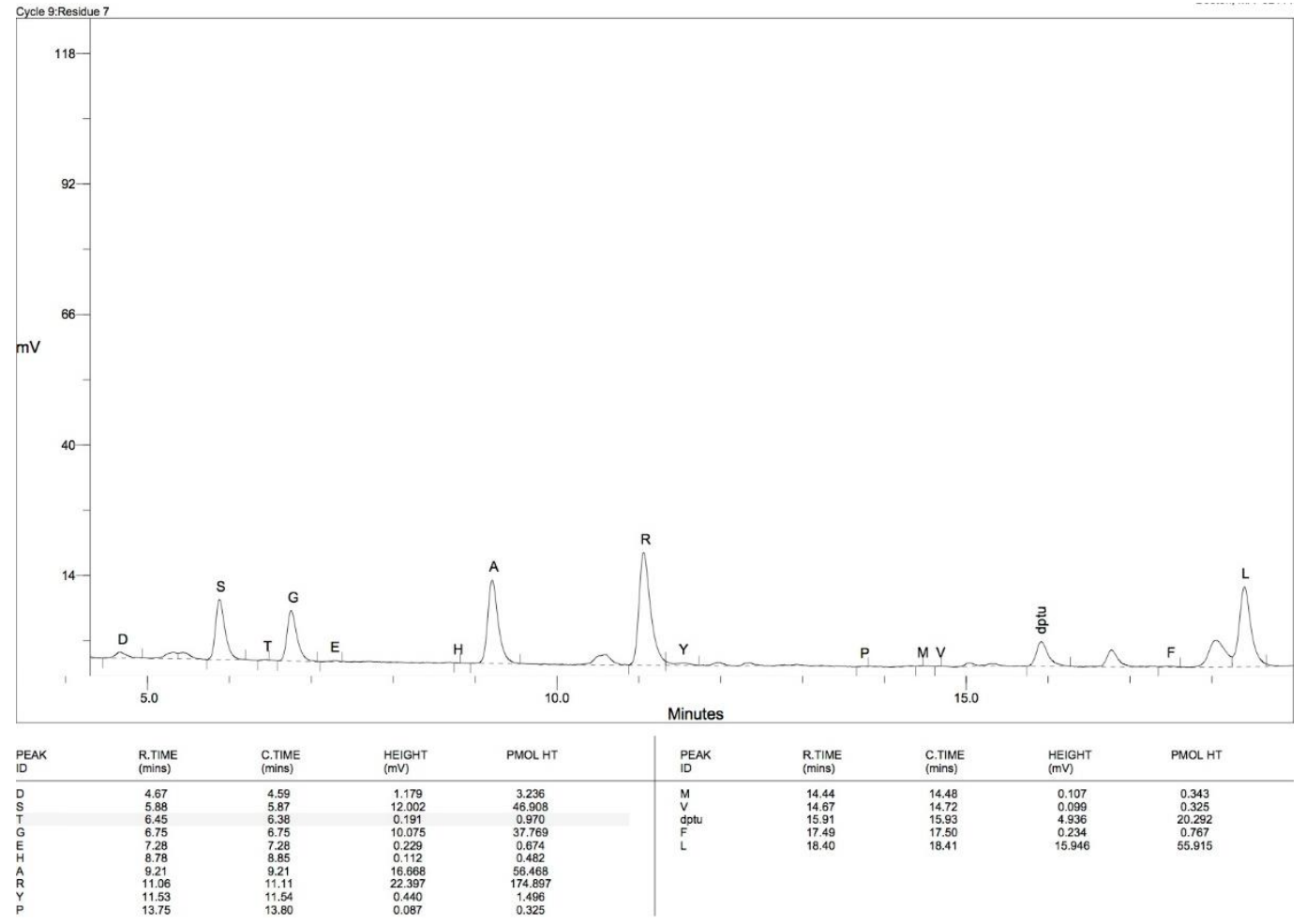

Figure S7: Edman Sequence Results for SL10 Amino Acid 7

The sequence of SL10 is R-L-D*-A-R-S-D*-G-B-B-R-M. D* is a fixed position in the library and is not recognized by Edman Degradation. 


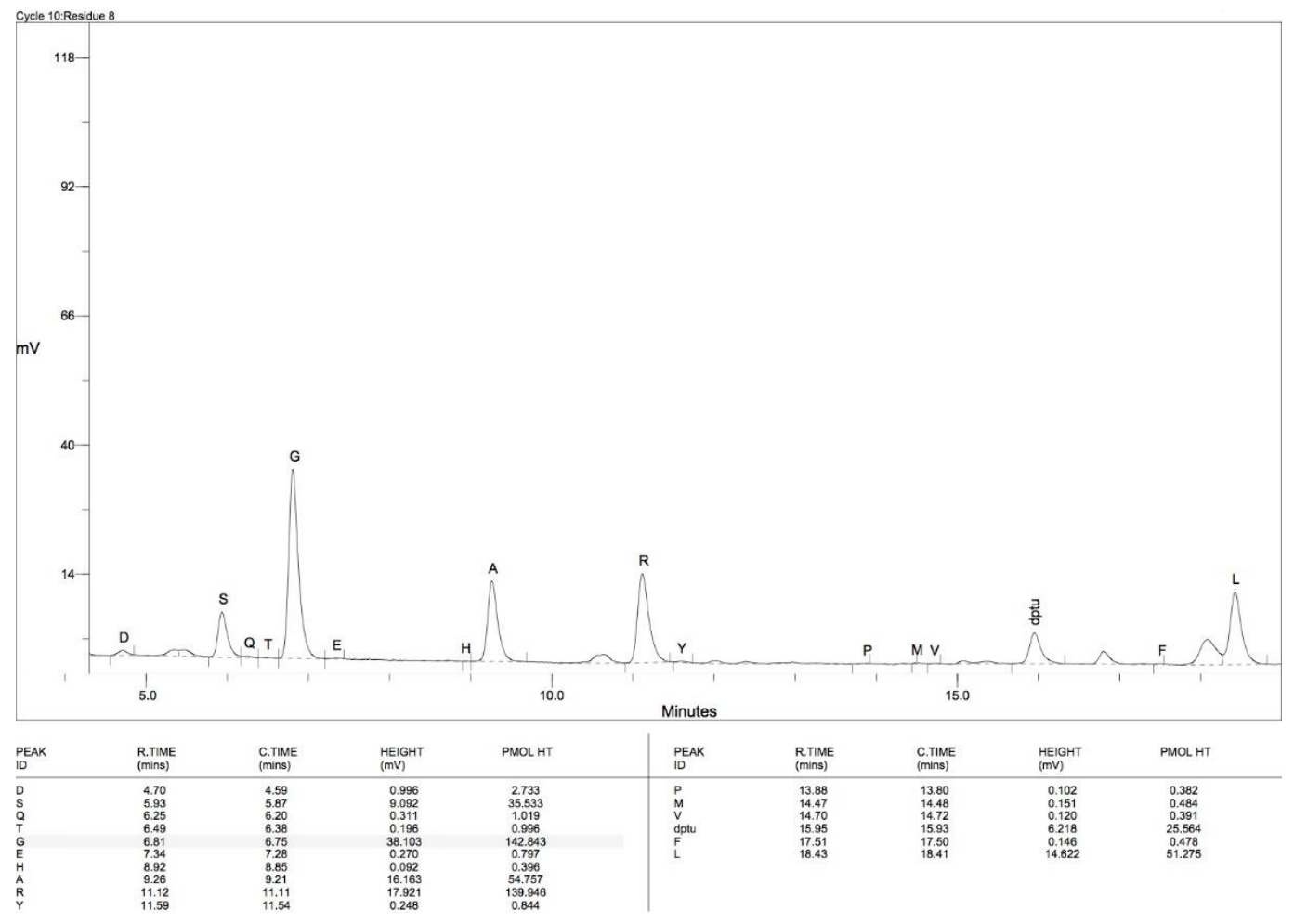

Figure S8: Edman Sequence Results for SL10 Amino Acid 8

The sequence of SL10 is R-L-D*-A-R-S-D*-G-B-B-R-M. Glycine was present in the fifth randomized position.

\section{Edman Degradation results for SL 11.}

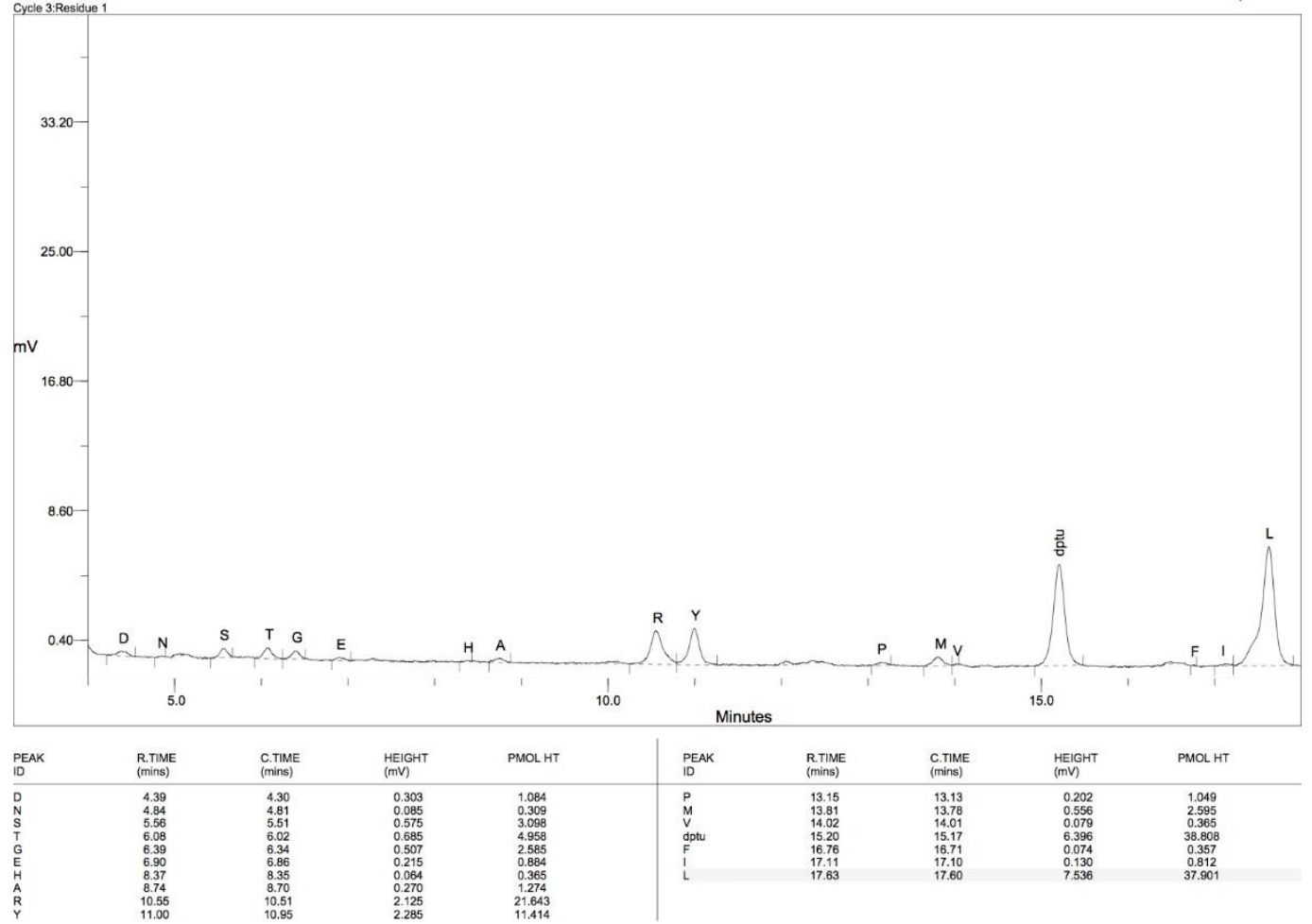

Figure S9: Edman Sequence Results for SL11 Amino Acid 1

The sequence of SL11 is R-L-D*-Y-L-T-D*-R-B-B-R-M. R is a fixed position in library. 


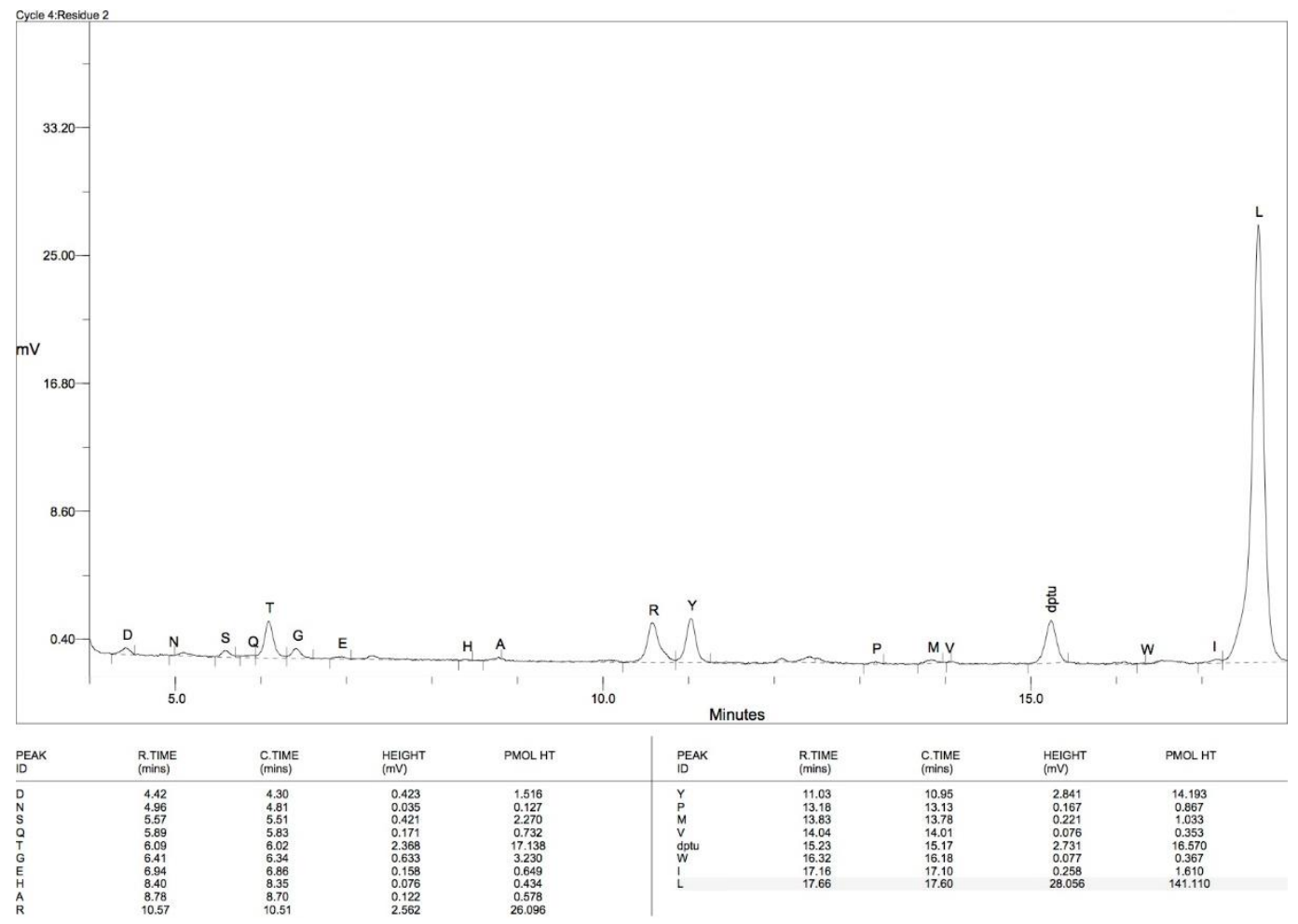

Figure S10: Edman Sequence Results for SL11 Amino Acid 2

The sequence of SL11 is R-L-D*-Y-L-T-D*-R-B-B-R-M. Leucine was present in the first randomized position.

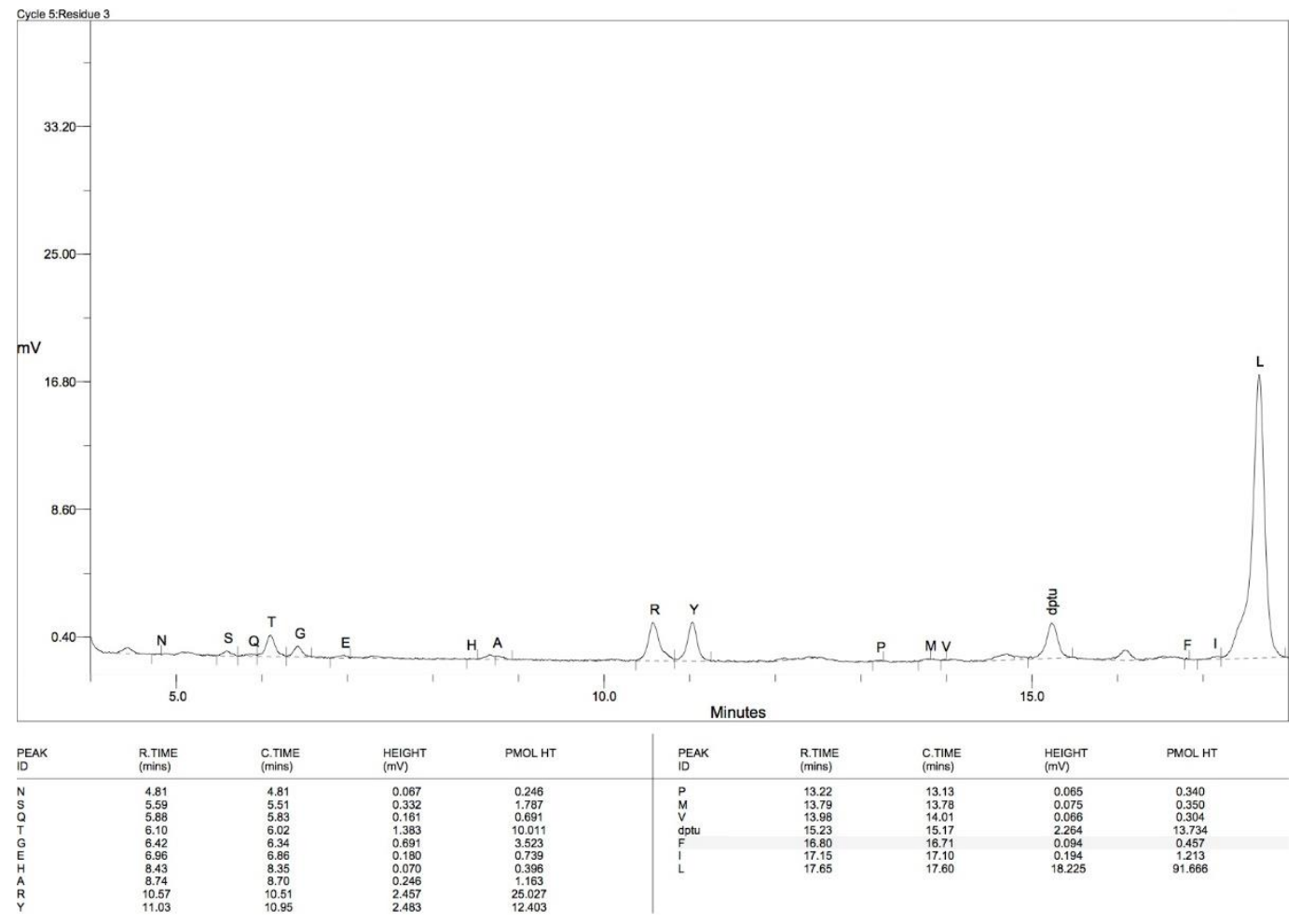

Figure S11: Edman Sequence Results for SL11 Amino Acid 3

The sequence of SL11 is R-L-D*-Y-L-T-D*-R-B-B-R-M. D* is a fixed position in the library and is not recognized by Edman Degradation. 


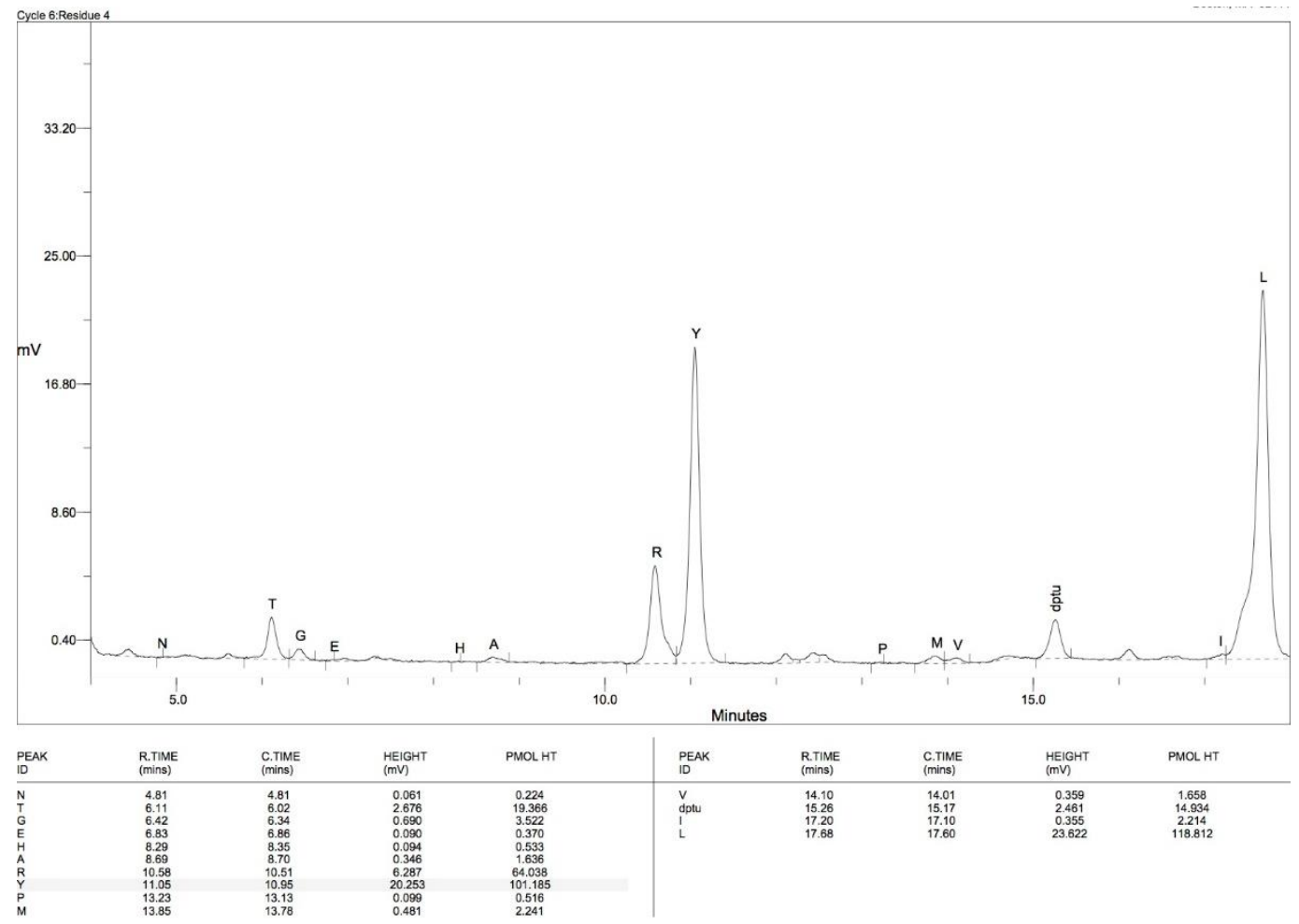

Figure S12: Edman Sequence Results for SL11 Amino Acid 4

The sequence of SL11 is R-L-D*-Y-L-T-D*-R-B-B-R-M. Tyrosine was present in the second randomized position.

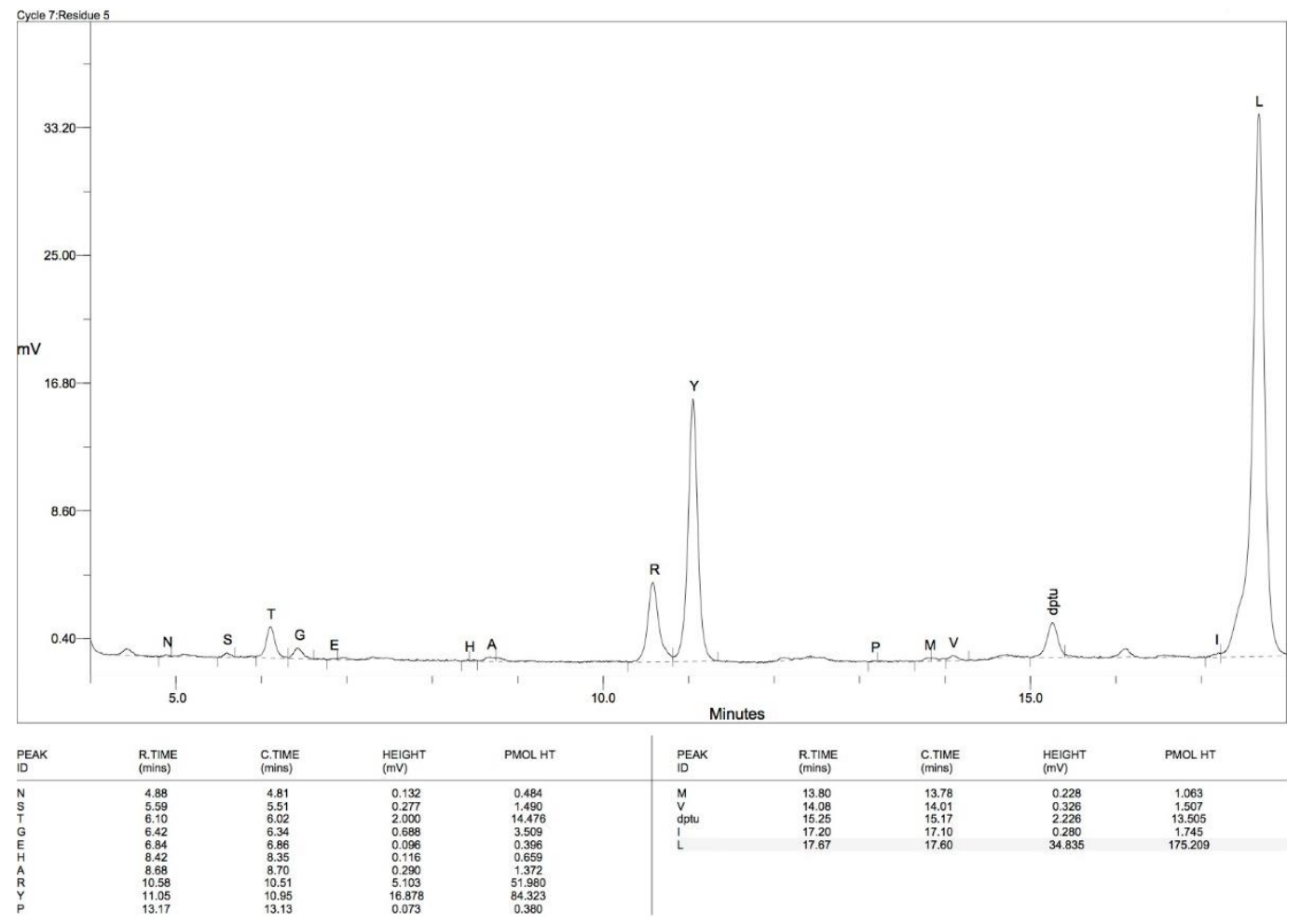

Figure S13: Edman Sequence Results for SL11 Amino Acid 5

The sequence of SL11 is R-L-D*-Y-L-T-D*-R-B-B-R-M. Leucine was present in the third randomized position. 


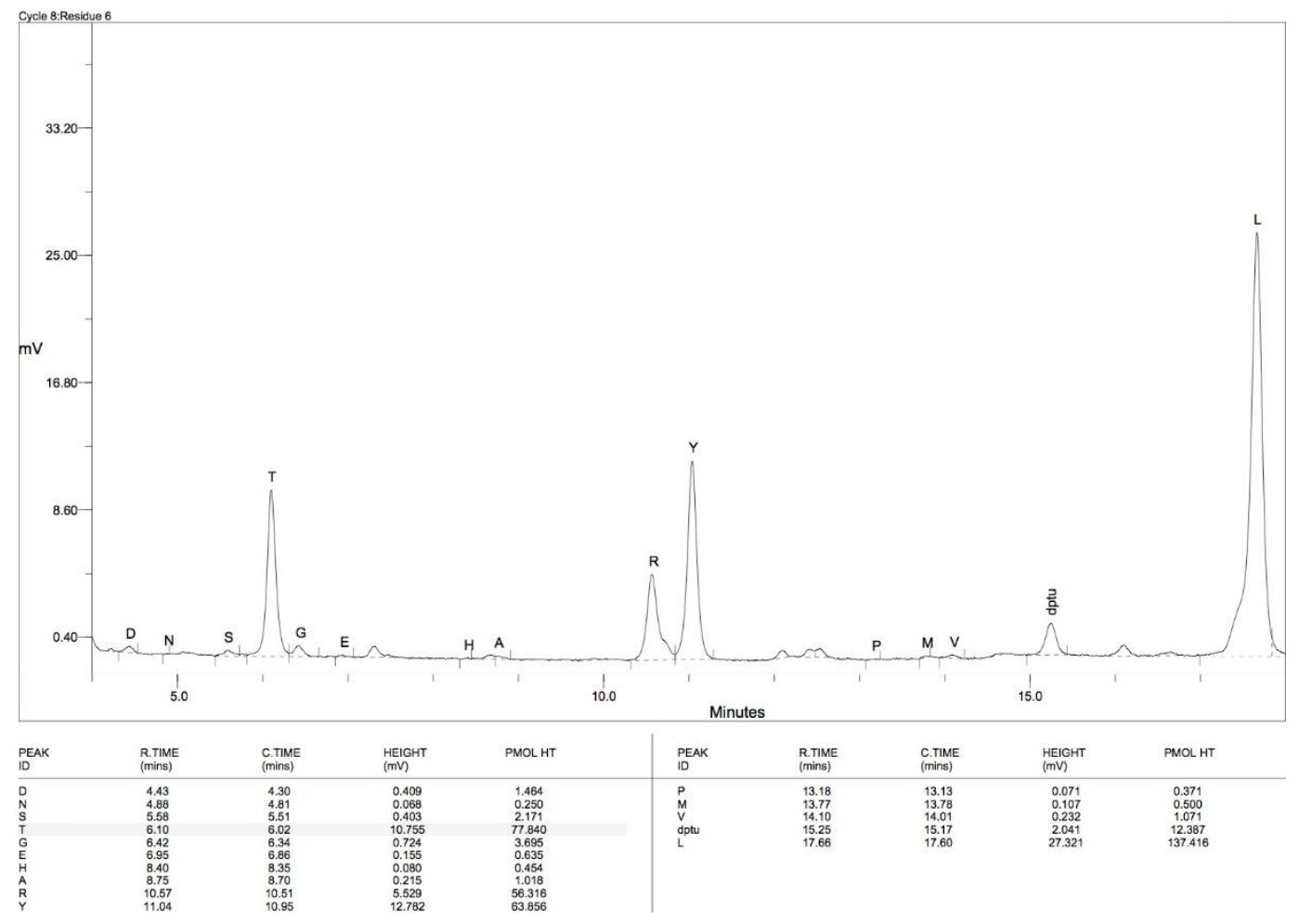

Figure S14: Edman Sequence Results for SL11 Amino Acid 6

The sequence of SL11 is R-L-D*-Y-L-T-D*-R-B-B-R-M. Threonine was present in the fourth randomized position.

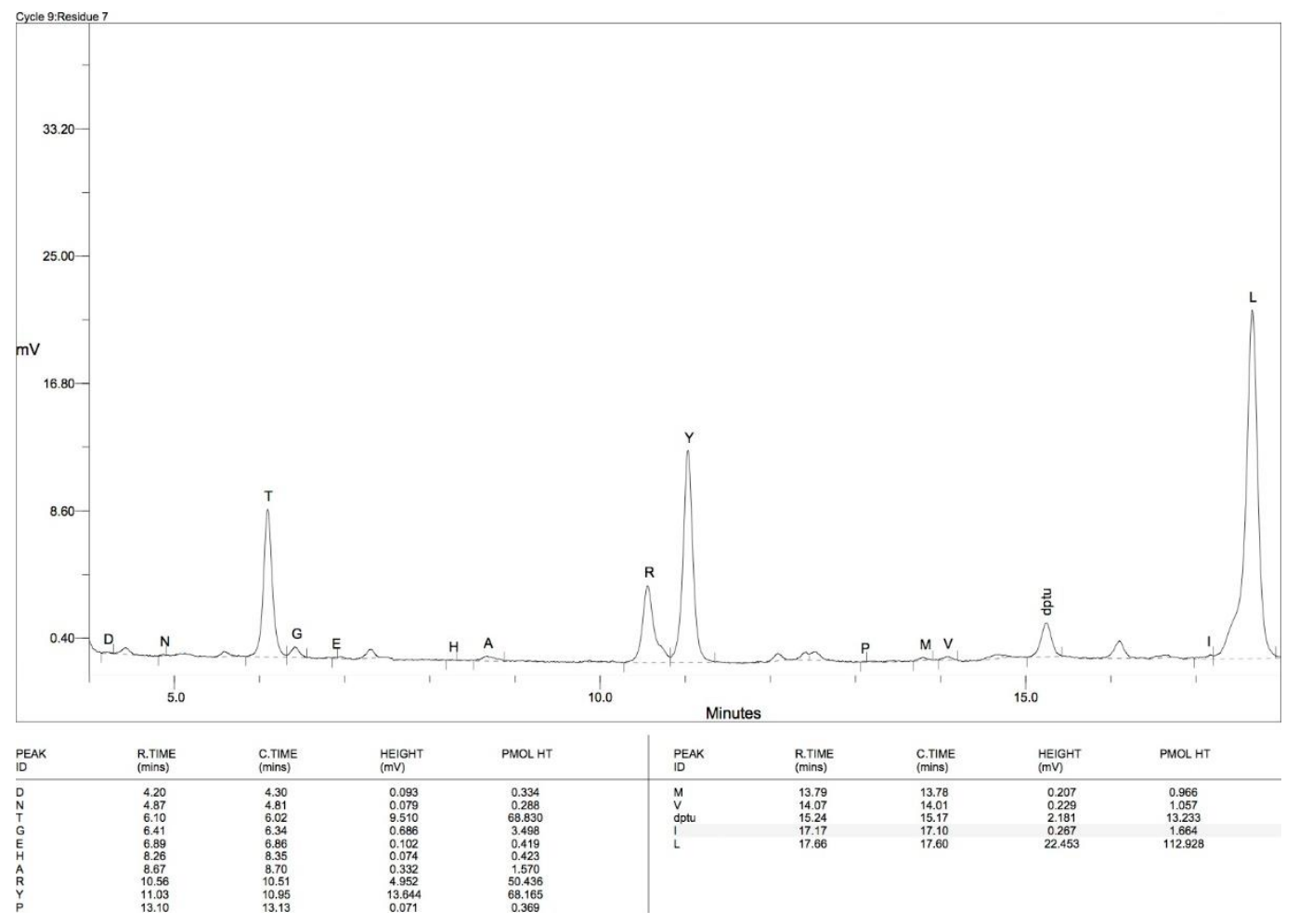

Figure S15: Edman Sequence Results for SL11 Amino Acid 7

The sequence of SL11 is R-L-D*-Y-L-T-D*-R-B-B-R-M. D* is a fixed position in the library and is not recognized by Edman Degradation. 


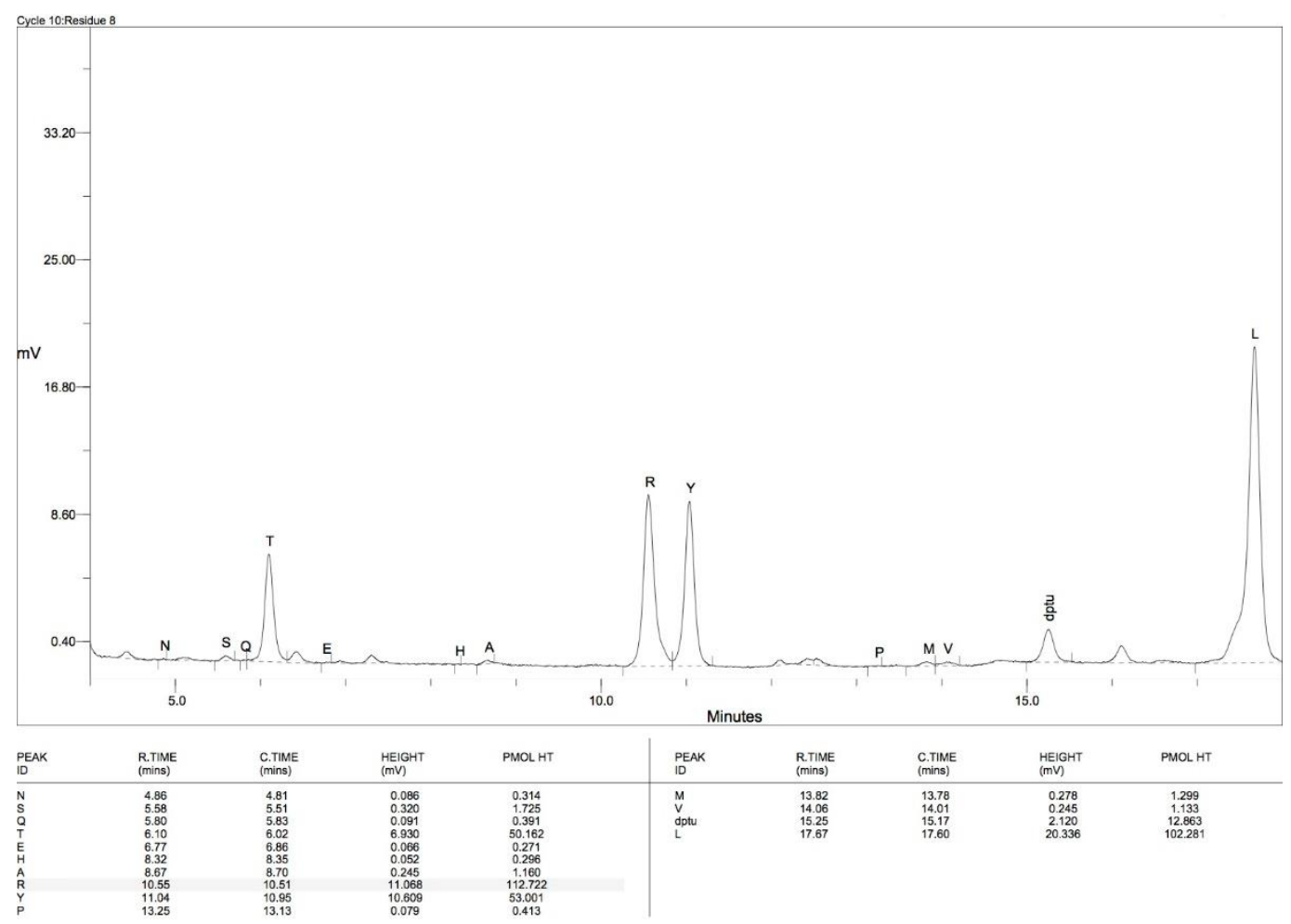

Figure S16: Edman Sequence Results for SL11 Amino Acid 8

The sequence of SL11 is R-L-D*-Y-L-T-D*-R-B-B-R-M. Arginine was present in the fifth randomized position.

\section{Edman Degradation results for SL 12.}

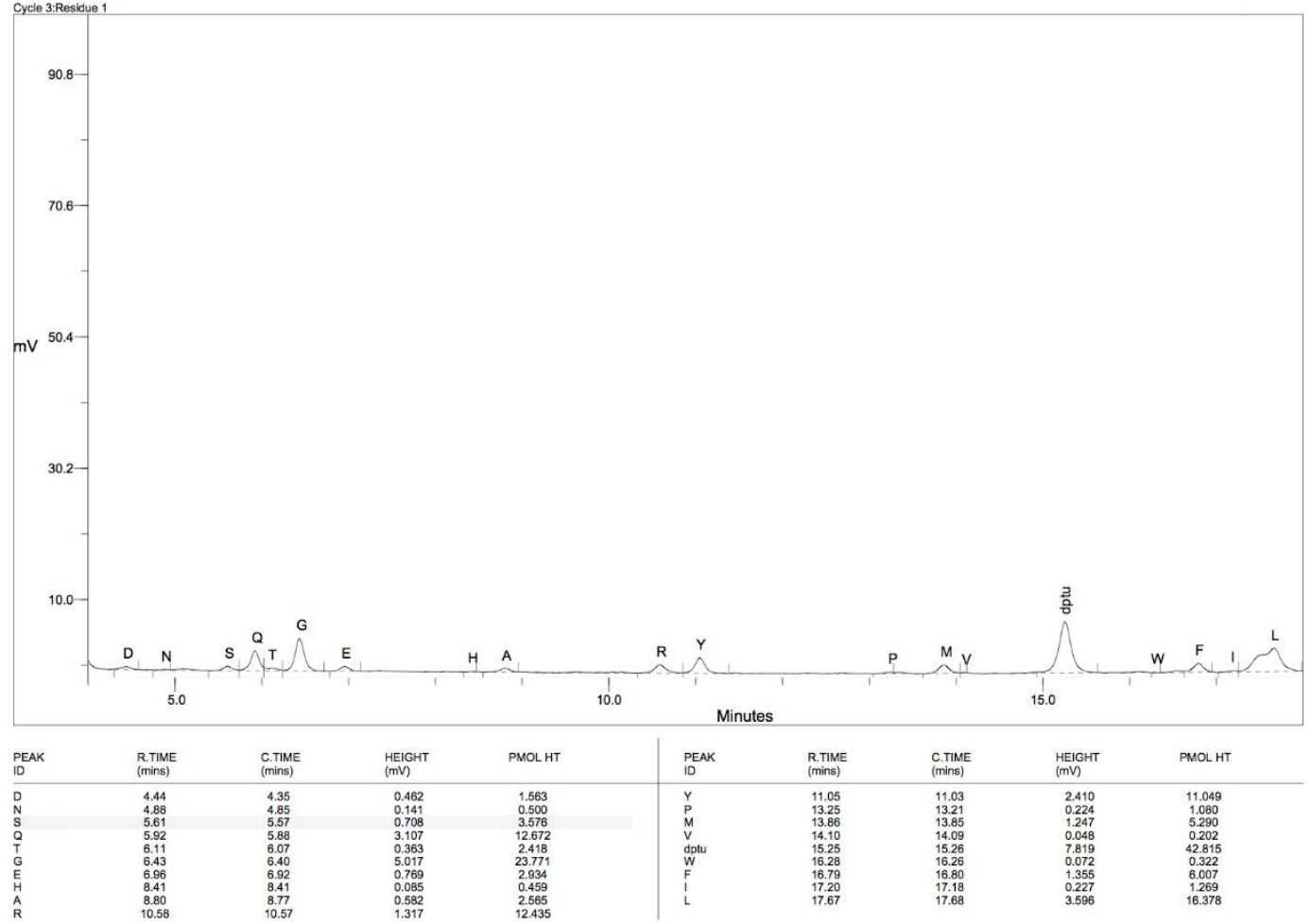

Figure S17: Edman Sequence Results for SL12 Amino Acid 1

The sequence of SL12 is R-L-D*-G-F-Y-D*-Q-B-B-R-M. R is a fixed position in library. 


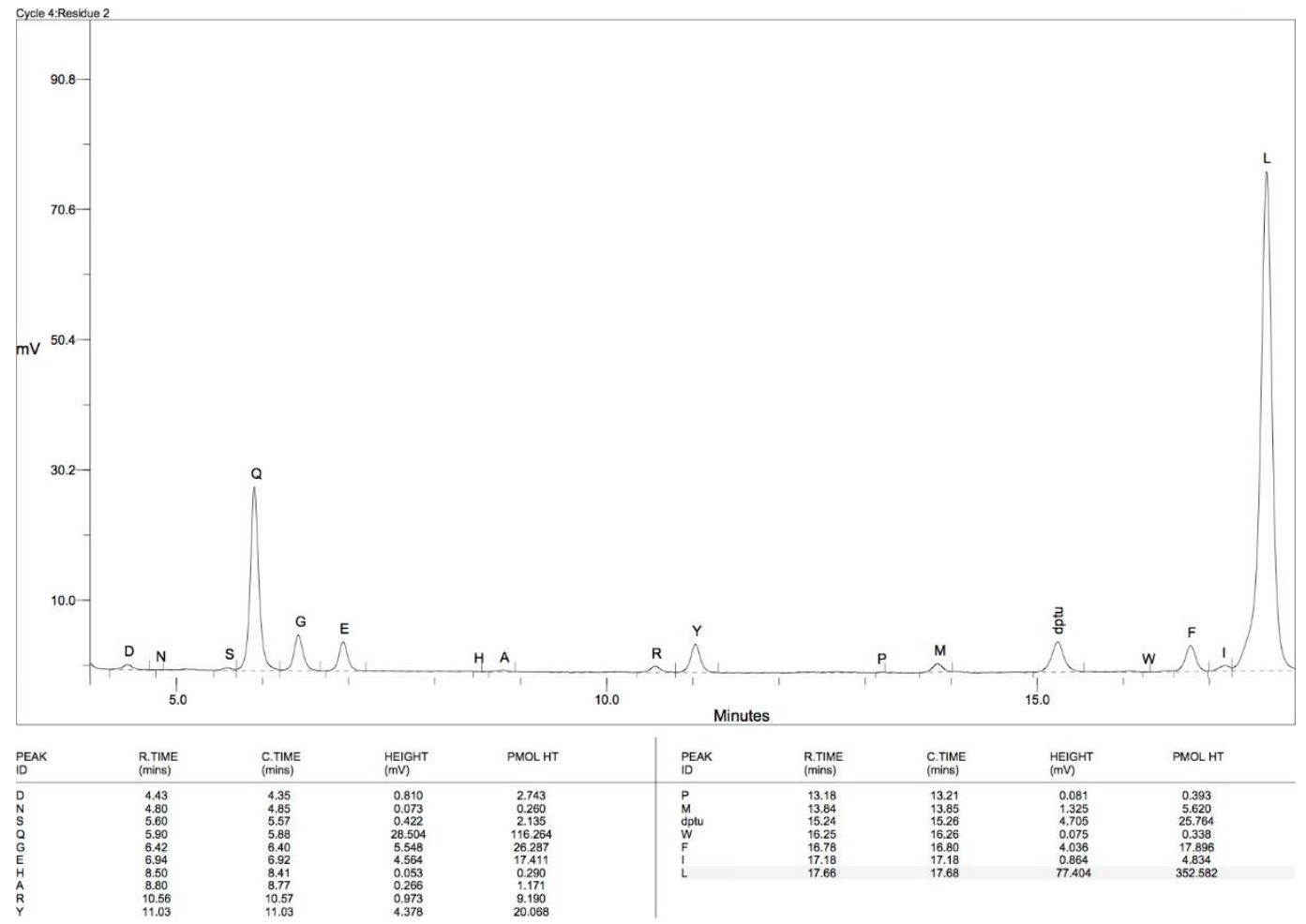

Figure S18: Edman Sequence Results for SL12 Amino Acid 2

The sequence of SL12 is R-L-D*-G-F-Y-D*-Q-B-B-R-M. Leucine was present in the first randomized position.

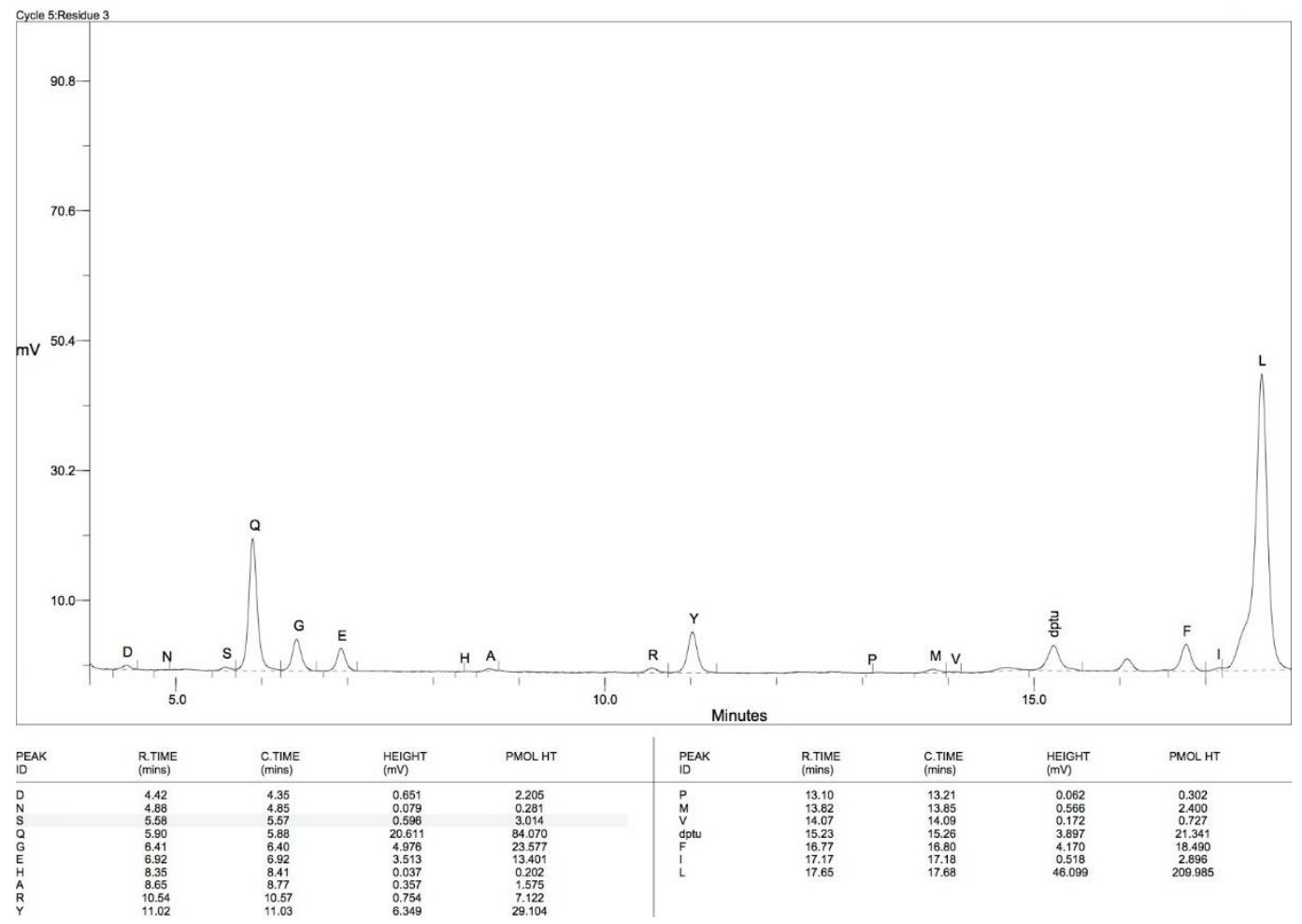

Figure S19: Edman Sequence Results for SL12 Amino Acid 3

The sequence of SL12 is R-L-D*-G-F-Y-D*-Q-B-B-R-M. D* is a fixed position in the library and is not recognized by Edman Degradation. 


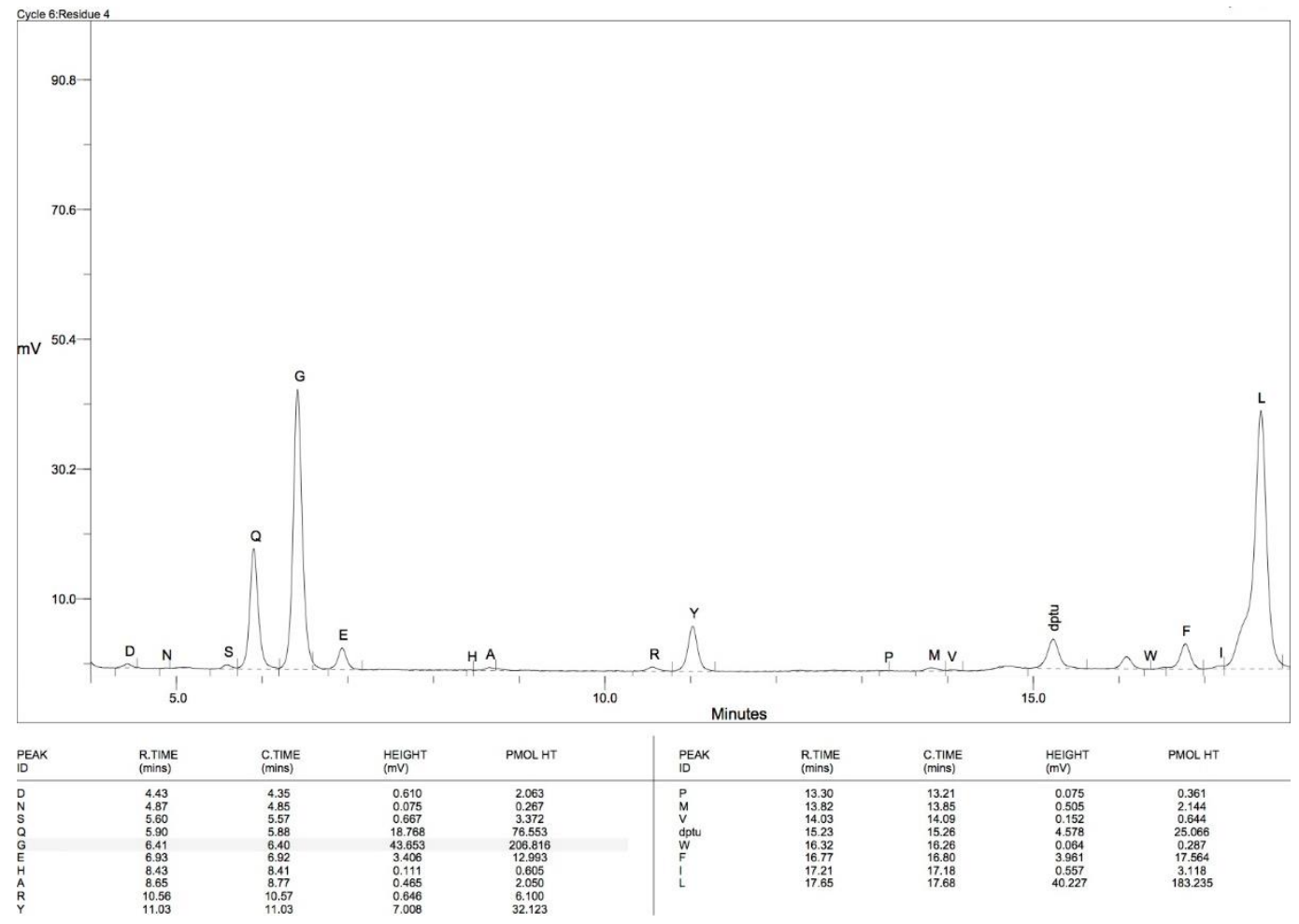

Figure S20: Edman Sequence Results for SL12 Amino Acid 4

The sequence of SL12 is R-L-D*-G-F-Y-D*-Q-B-B-R-M. Glycine was present in the second randomized position.

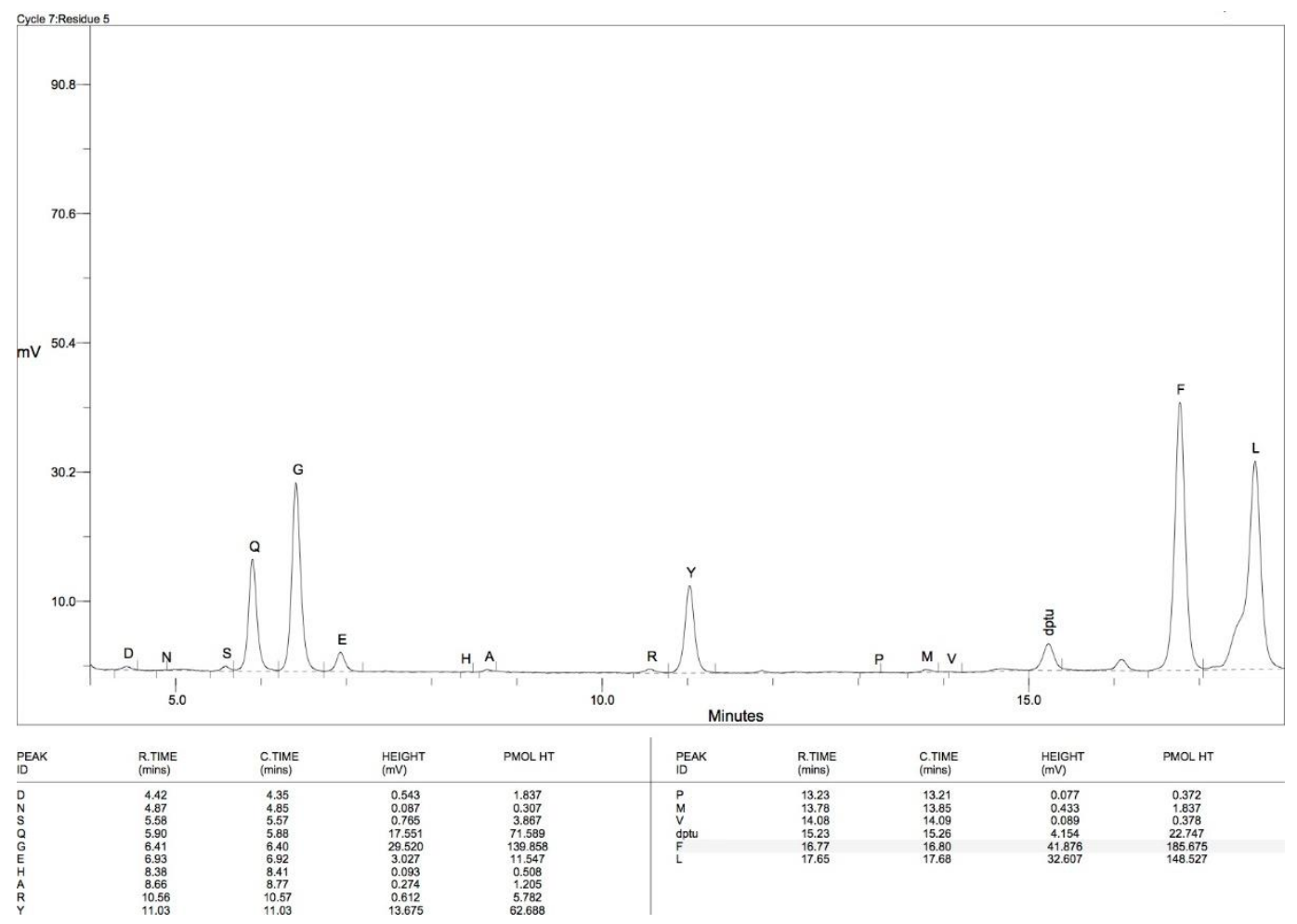

Figure S21: Edman Sequence Results for SL12 Amino Acid 5

The sequence of SL12 is R-L-D*-G-F-Y-D*-Q-B-B-R-M. Phenylalanine was present in the third randomized position. 


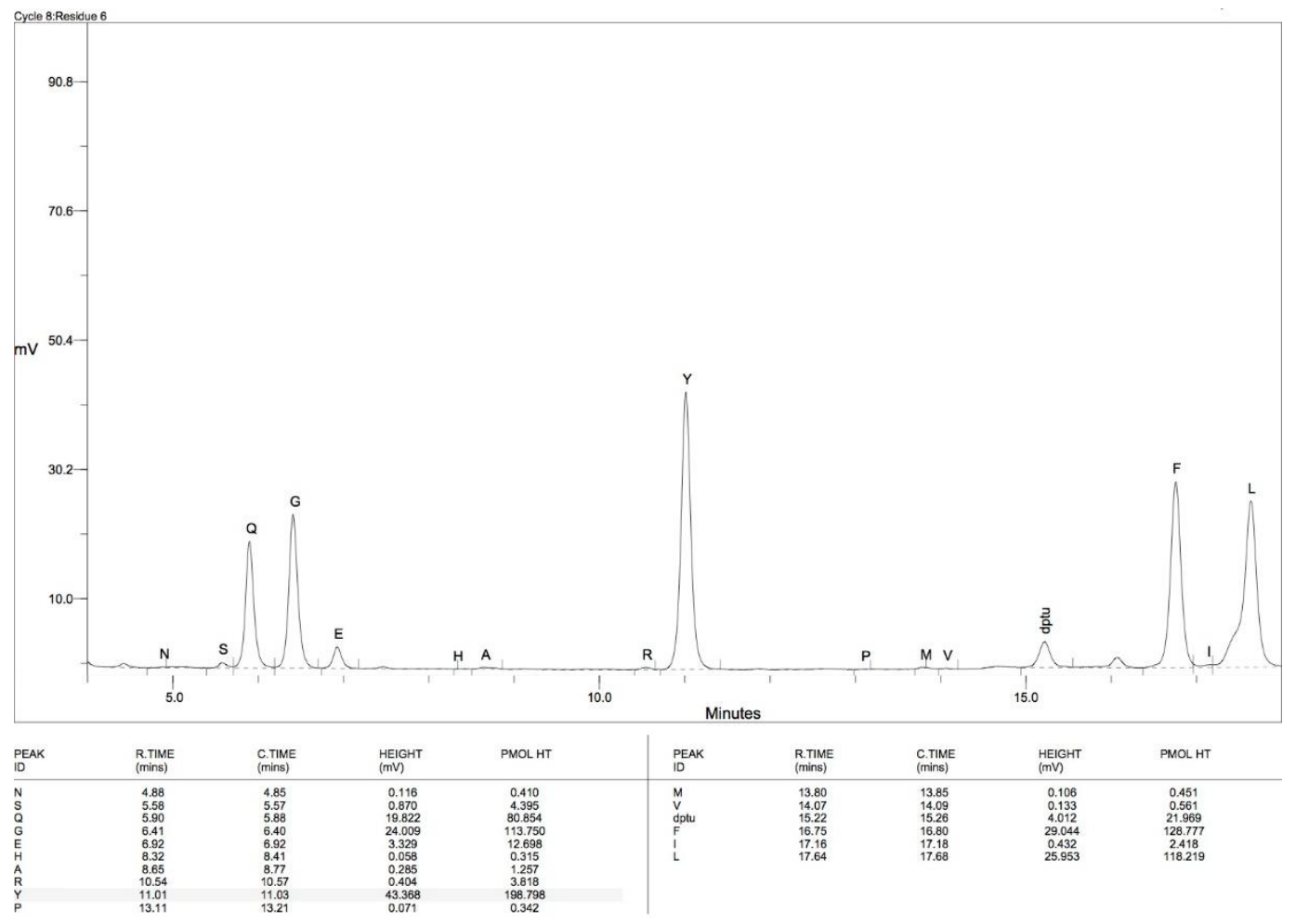

Figure S21: Edman Sequence Results for SL12 Amino Acid 6

The sequence of SL12 is R-L-D*-G-F-Y-D*-Q-B-B-R-M. Tyrosine was present in the fourth randomized position.

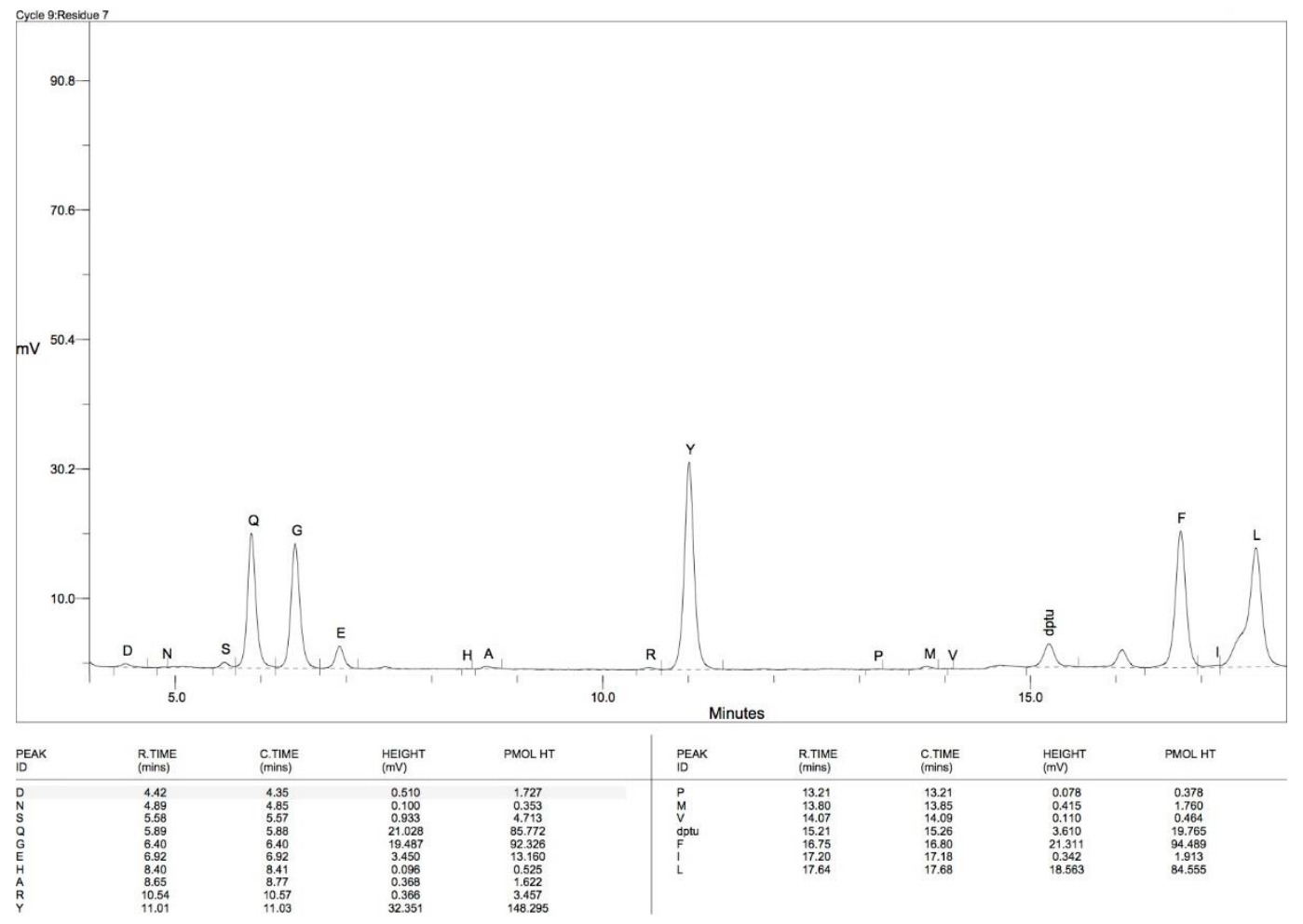

Figure S22: Edman Sequence Results for SL12 Amino Acid 7

The sequence of SL12 is R-L-D*-G-F-Y-D*-Q-B-B-R-M. D* is a fixed position in the library and is not recognized by Edman Degradation. 


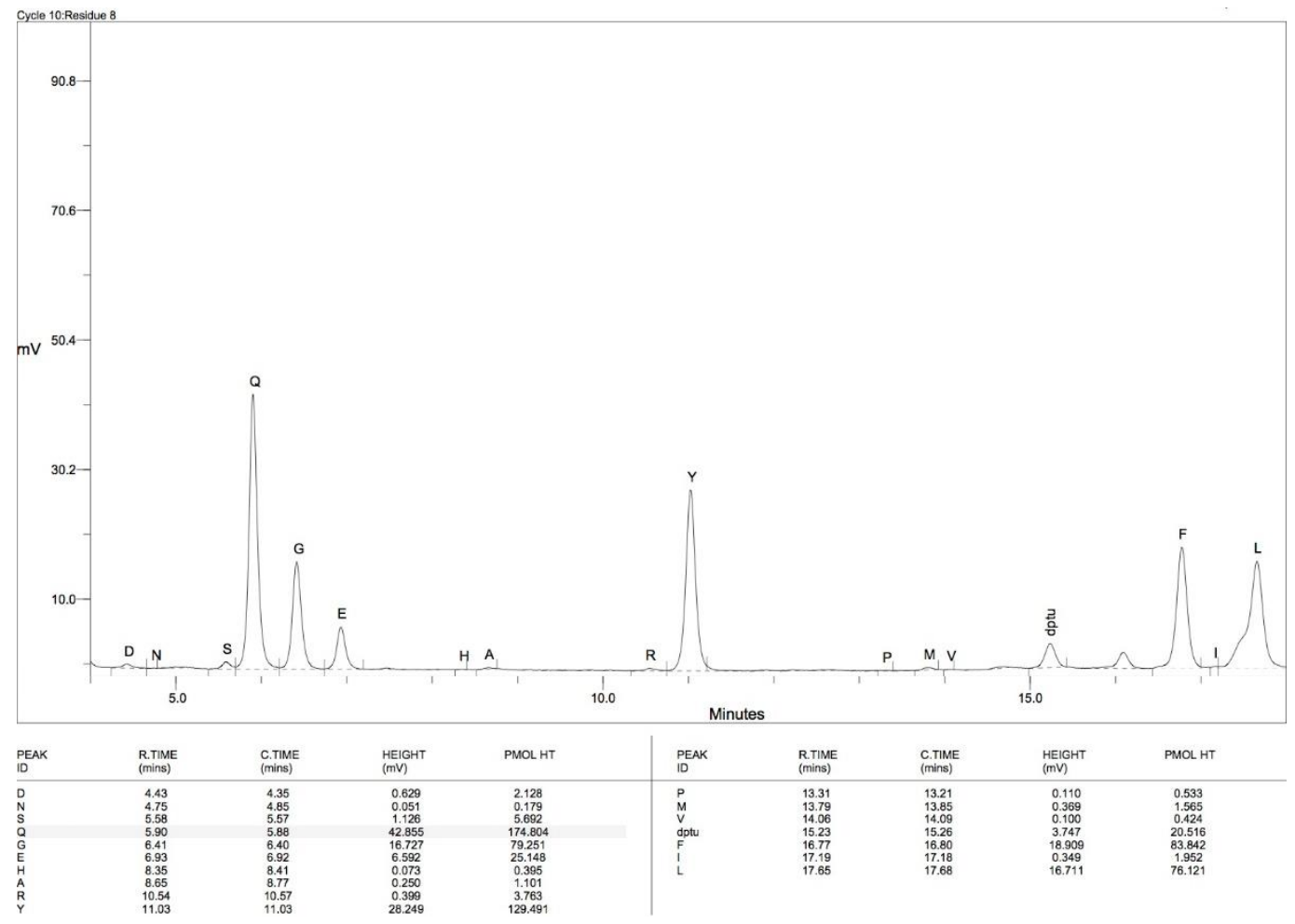

Figure S23: Edman Sequence Results for SL12 Amino Acid 8

The sequence of SL12 is R-L-D*-G-F-Y-D*-Q-B-B-R-M. Glutamine was present in the fifth randomized position.

\section{Edman Degradation results for SL 13.}

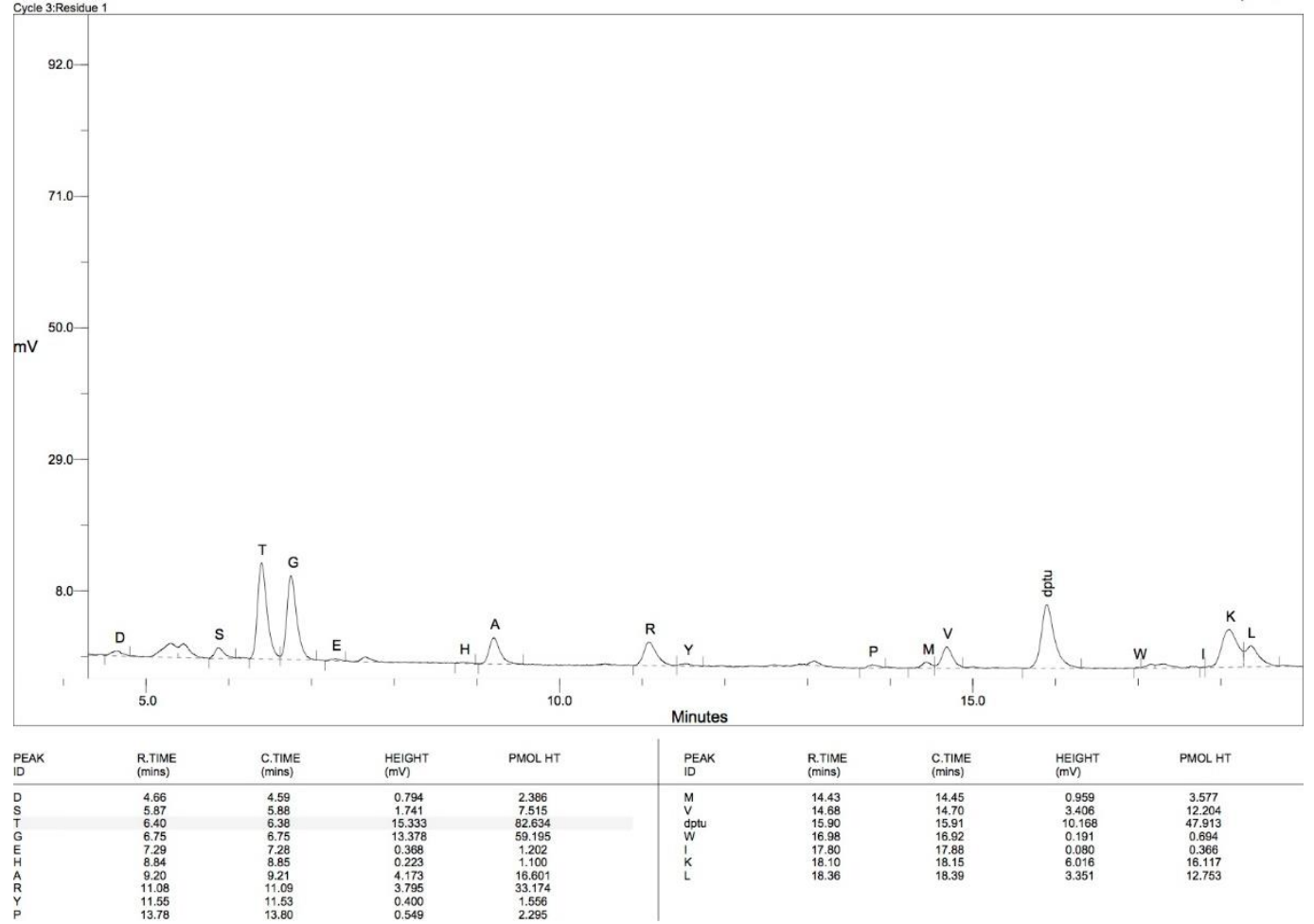

Figure S24: Edman Sequence Results for SL13 Amino Acid 1

The sequence of SL13 is R-T-D*-G-L-A-D*-V-B-B-R-M. $R$ is a fixed position in library. 


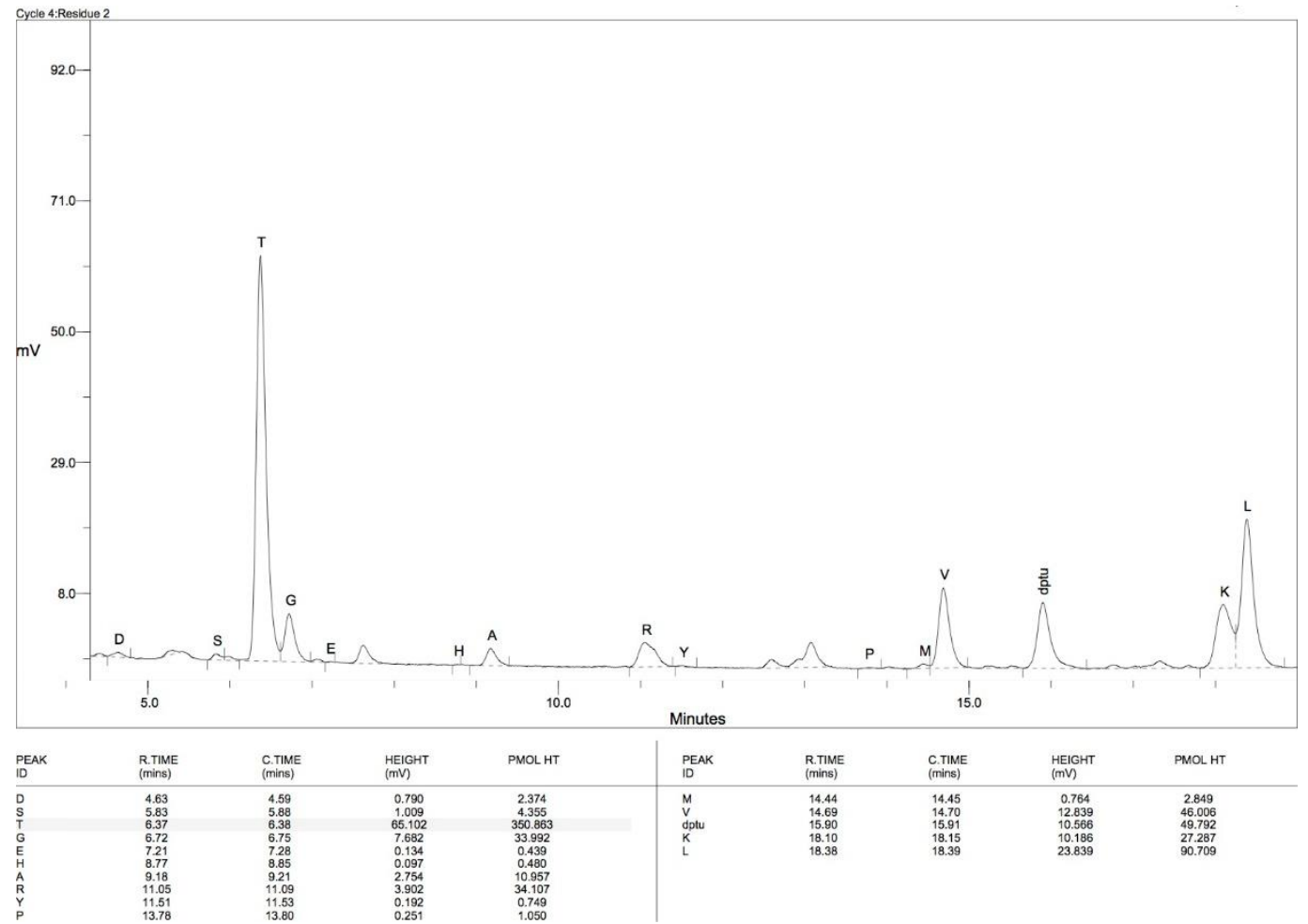

Figure S25: Edman Sequence Results for SL13 Amino Acid 2

The sequence of SL13 is R-T-D*-G-L-A-D*-V-B-B-R-M. Threonine was present in the first randomized position.

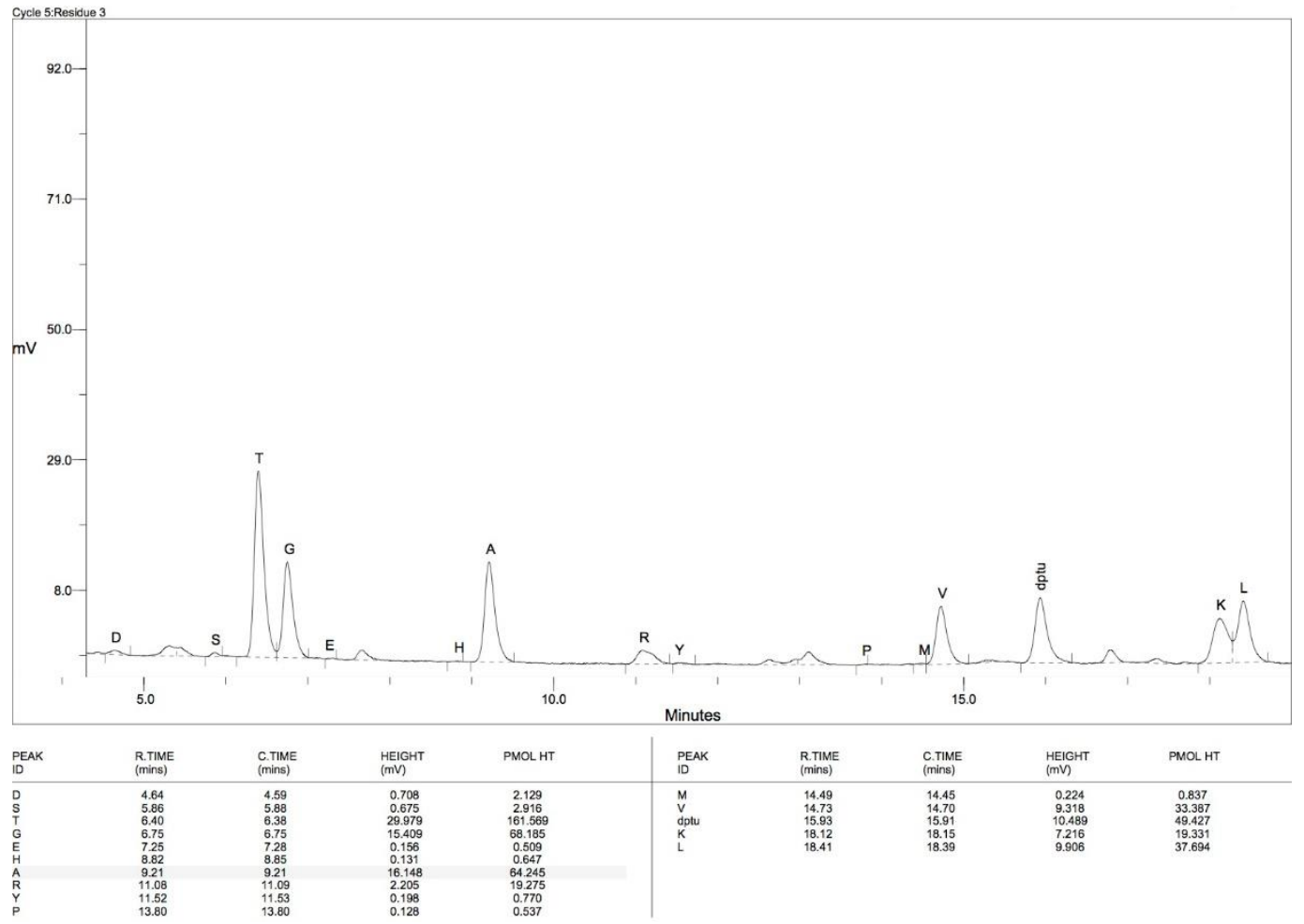

Figure S26: Edman Sequence Results for SL13 Amino Acid 3

The sequence of SL13 is R-T-D*-G-L-A-D*-V-B-B-R-M. D* is a fixed position in the library and is not recognized by Edman Degradation. 


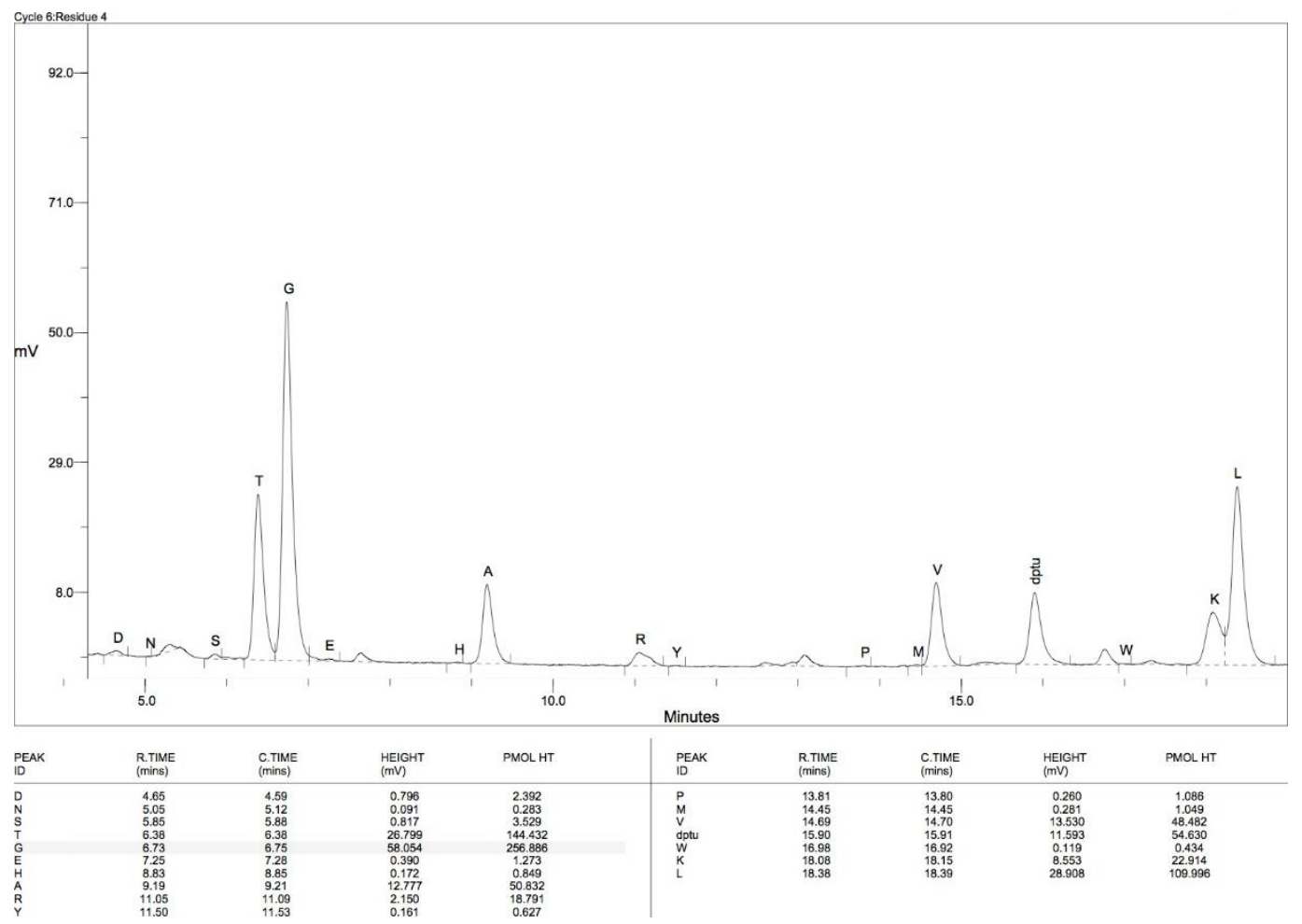

Figure S27: Edman Sequence Results for SL13 Amino Acid 4

The sequence of SL13 is R-T-D*-G-L-A-D*-V-B-B-R-M. Glycine was present in the second randomized position.

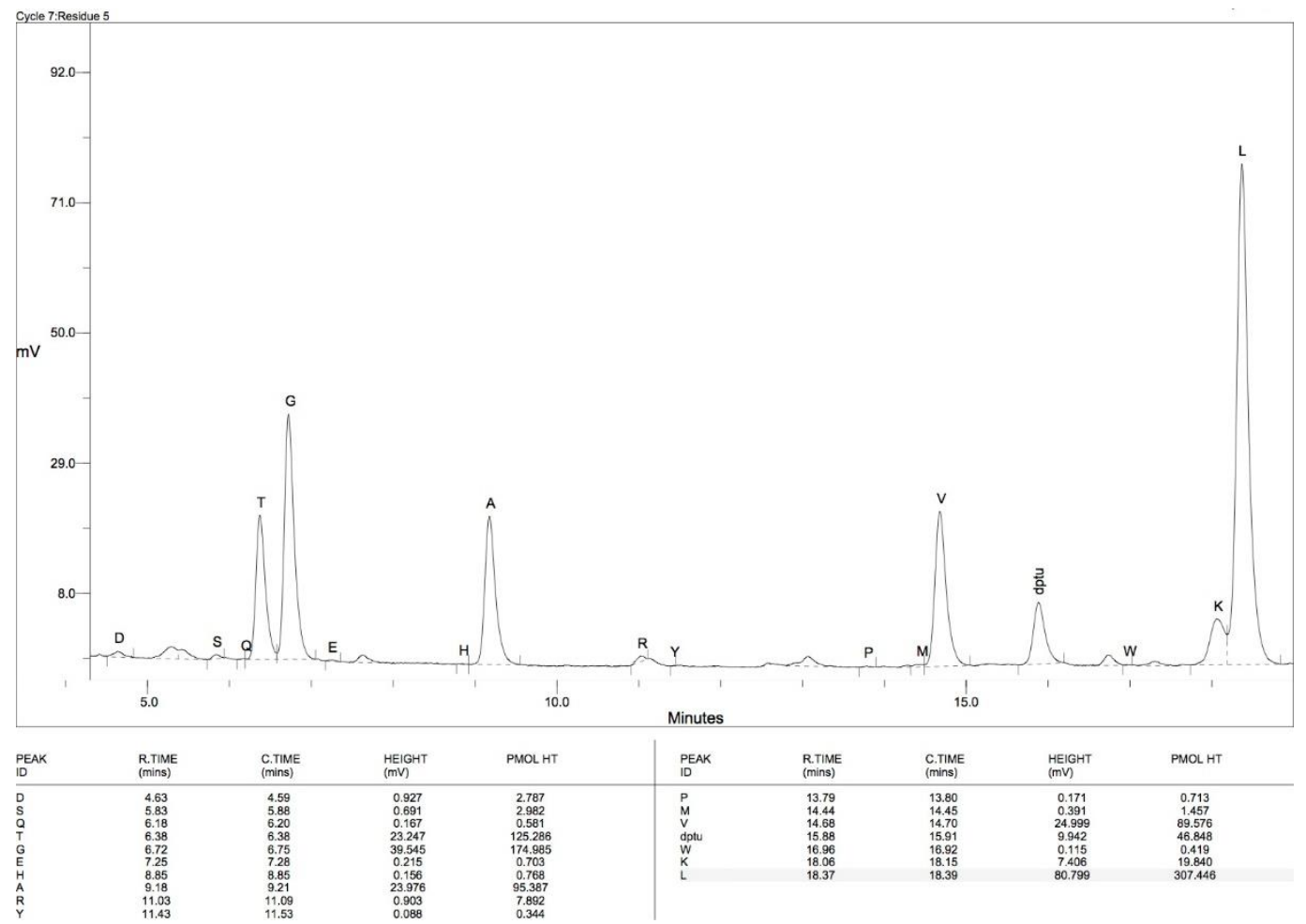

Figure S28: Edman Sequence Results for SL13 Amino Acid 5

The sequence of SL13 is R-T-D*-G-L-A-D*-V-B-B-R-M. Leucine was present in the third randomized position. 


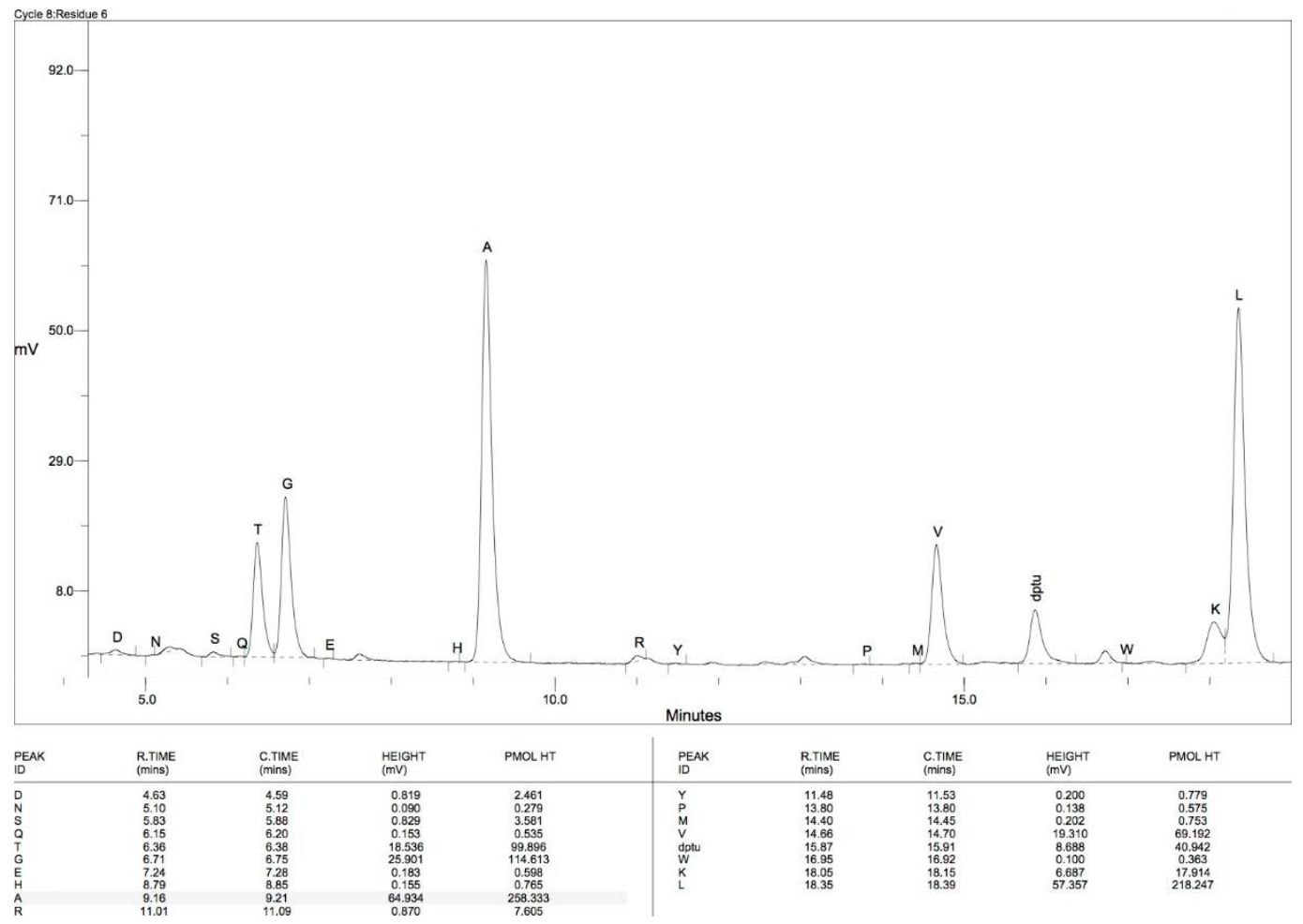

Figure S29: Edman Sequence Results for SL13 Amino Acid 6

The sequence of SL13 is R-T-D*-G-L-A-D*-V-B-B-R-M. Alanine was present in the fourth randomized position.
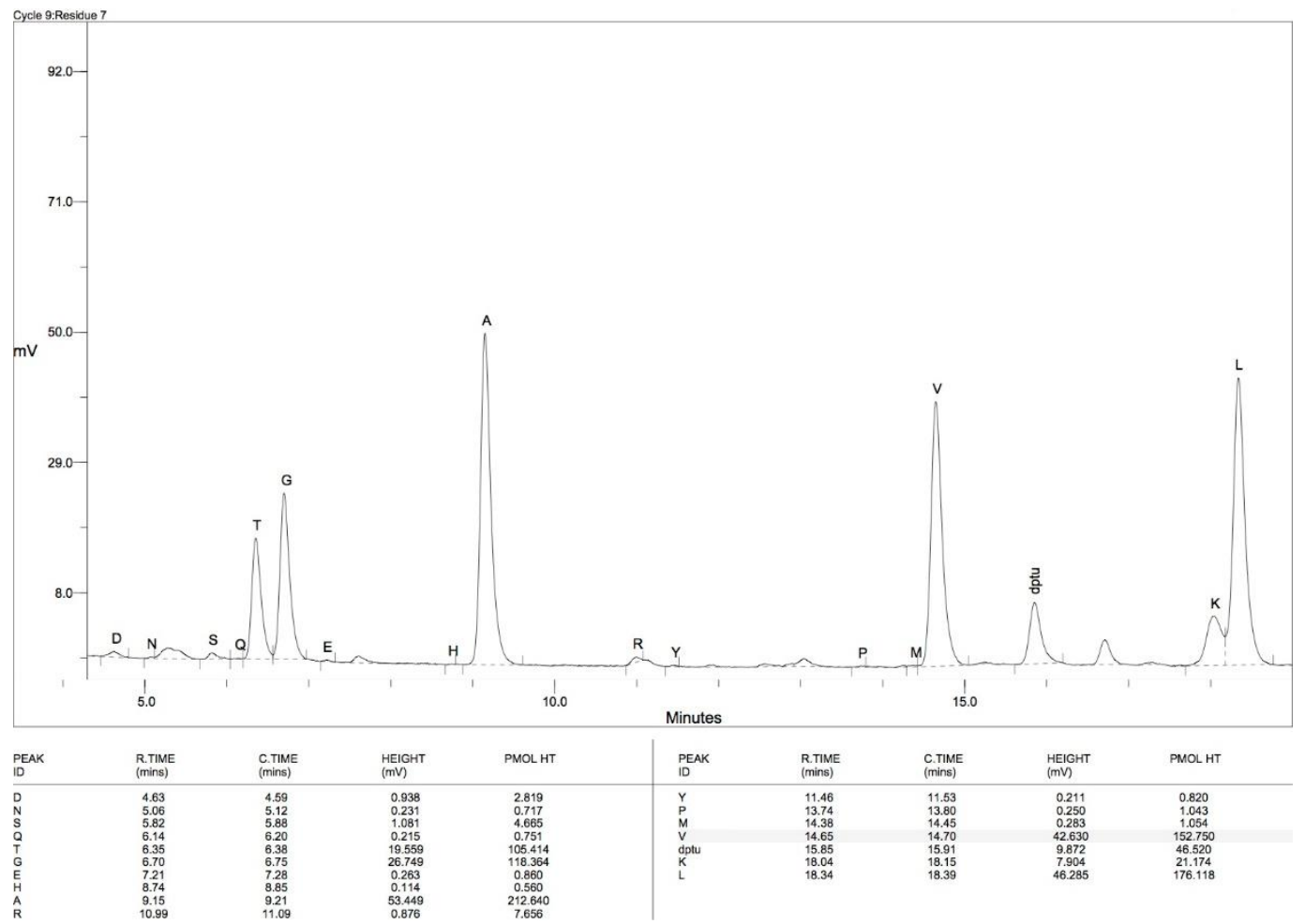

Figure S30: Edman Sequence Results for SL13 Amino Acid 7

The sequence of SL13 is R-T-D*-G-L-A-D*-V-B-B-R-M. D* is a fixed position in the library and is not recognized by Edman Degradation. 


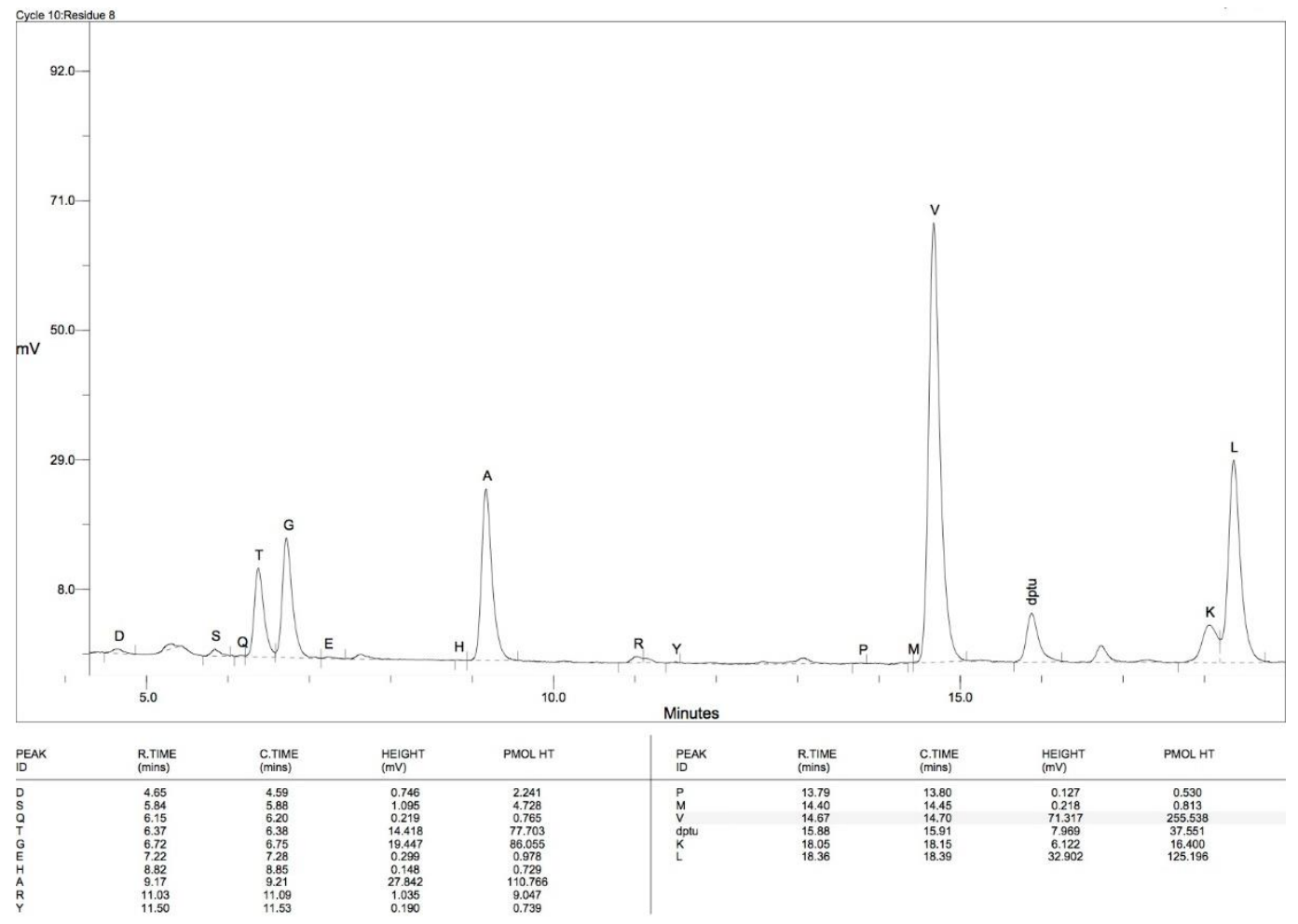

Figure S31: Edman Sequence Results for SL13 Amino Acid 8

The sequence of SL13 is R-T-D*-G-L-A-D*-V-B-B-R-M. Valine was present in the fifth randomized position.

\section{Edman Degradation results for SL 14.}

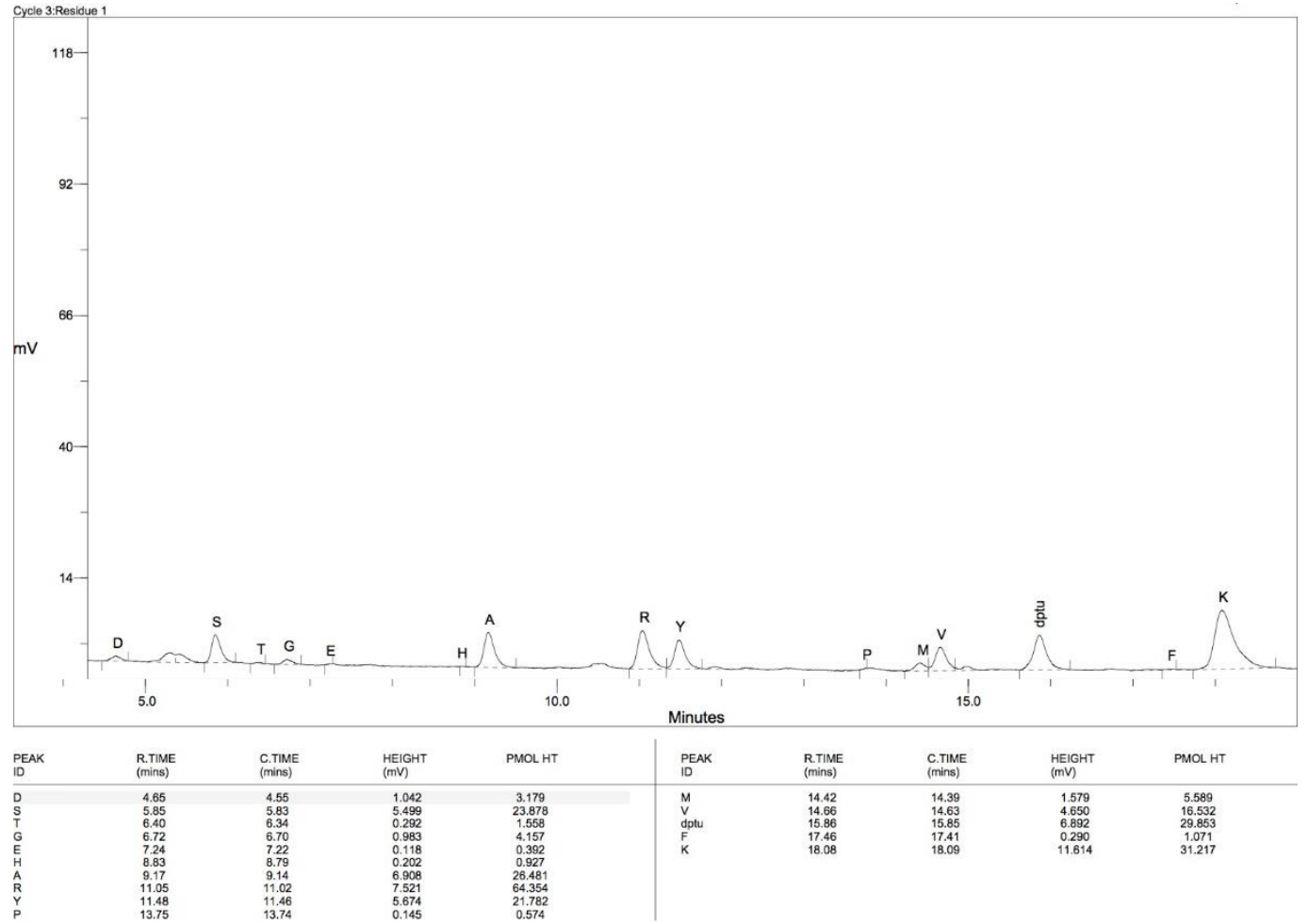

Figure S32: Edman Sequence Results for SL14 Amino Acid 1

The sequence of SL14 is R-Y-D*-R-A-S-D*-V-B-B-R-M. R is a fixed position in library. 


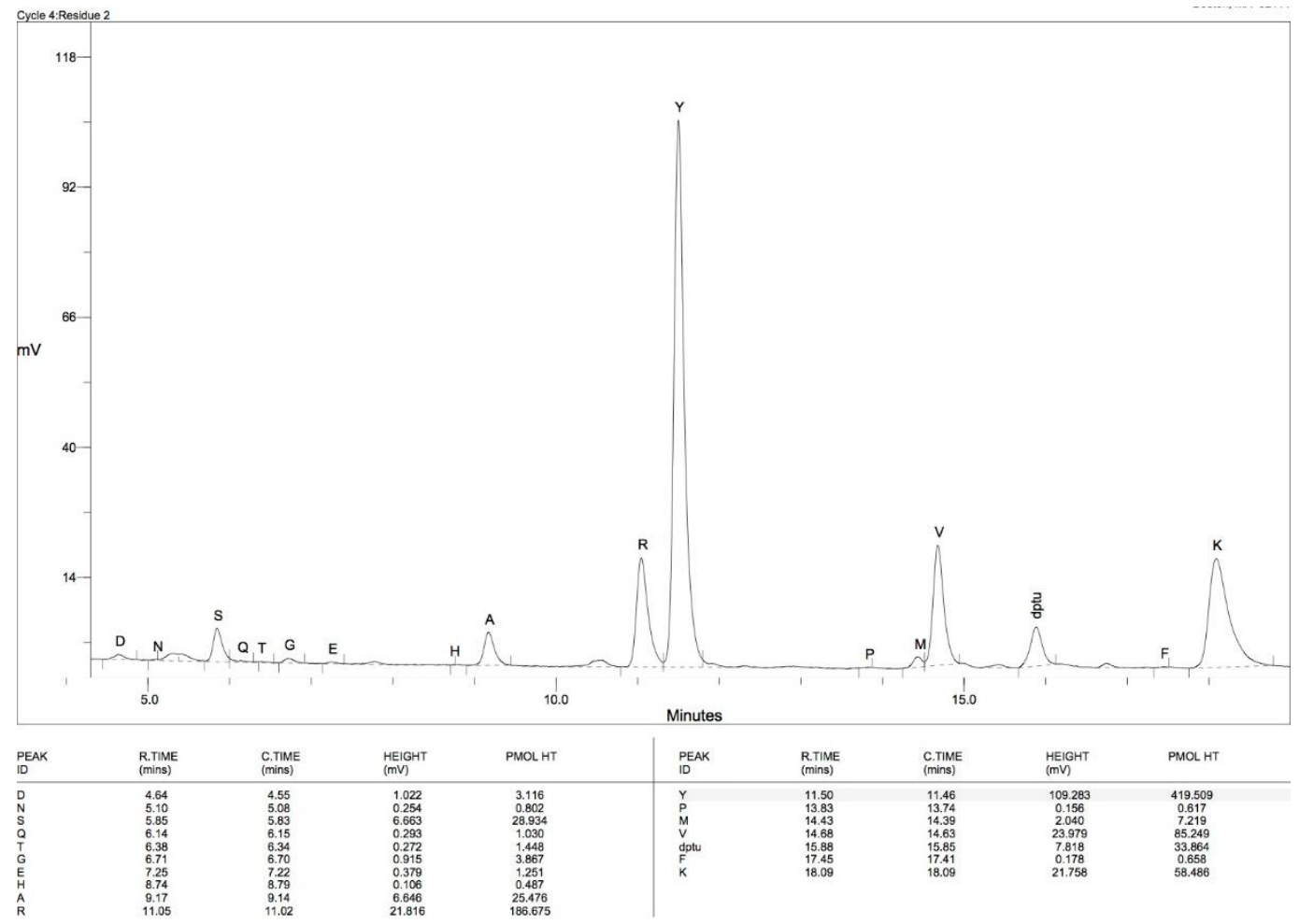

Figure S33: Edman Sequence Results for SL14 Amino Acid 2

The sequence of SL14 is R-Y-D*-R-A-S-D*-V-B-B-R-M. Tyrosine was present in the first randomized position.

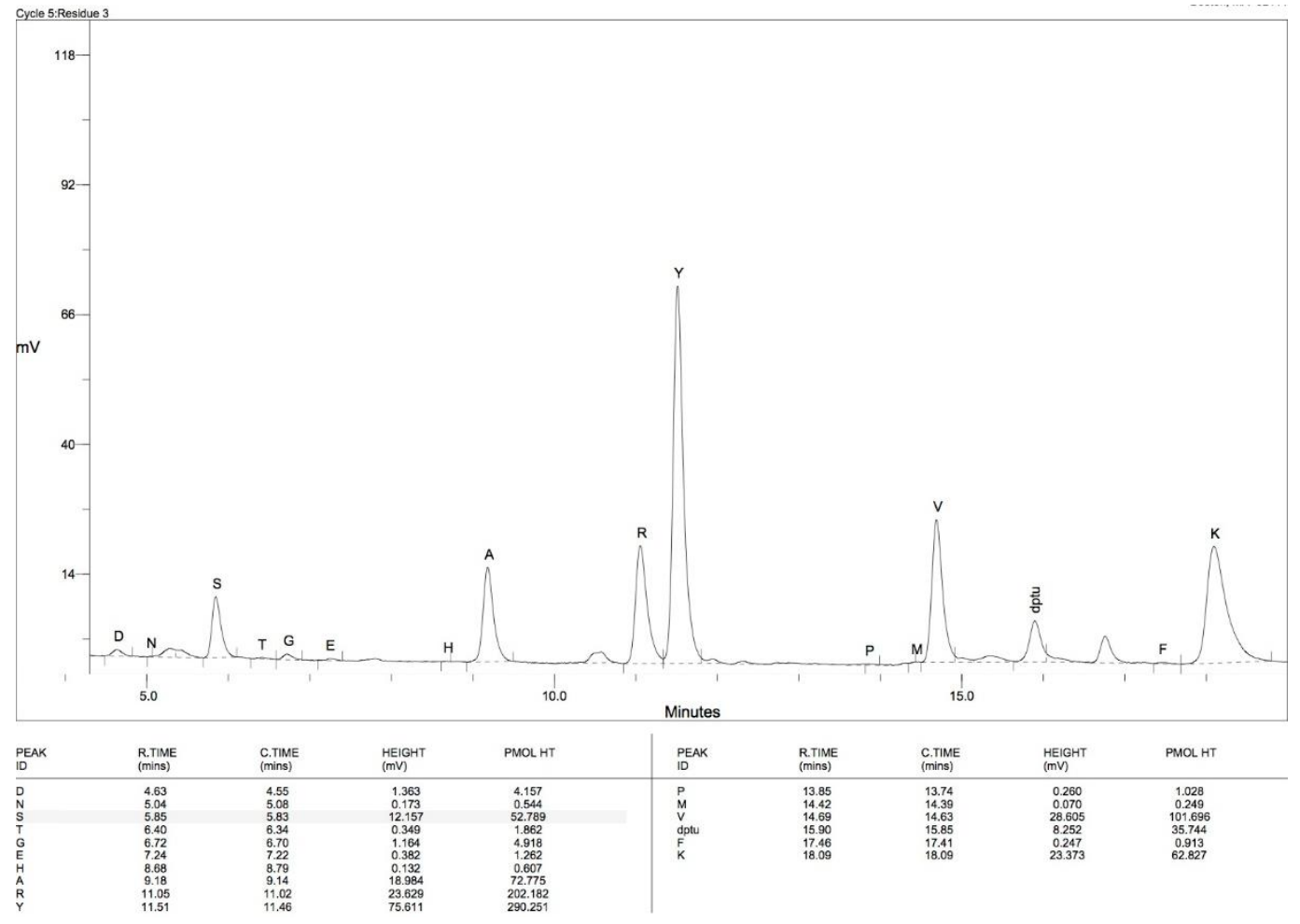

Figure S34: Edman Sequence Results for SL14 Amino Acid 3

The sequence of SL14 is R-Y-D*-R-A-S-D*-V-B-B-R-M. D* is a fixed position in the library and is not recognized by Edman Degradation. 


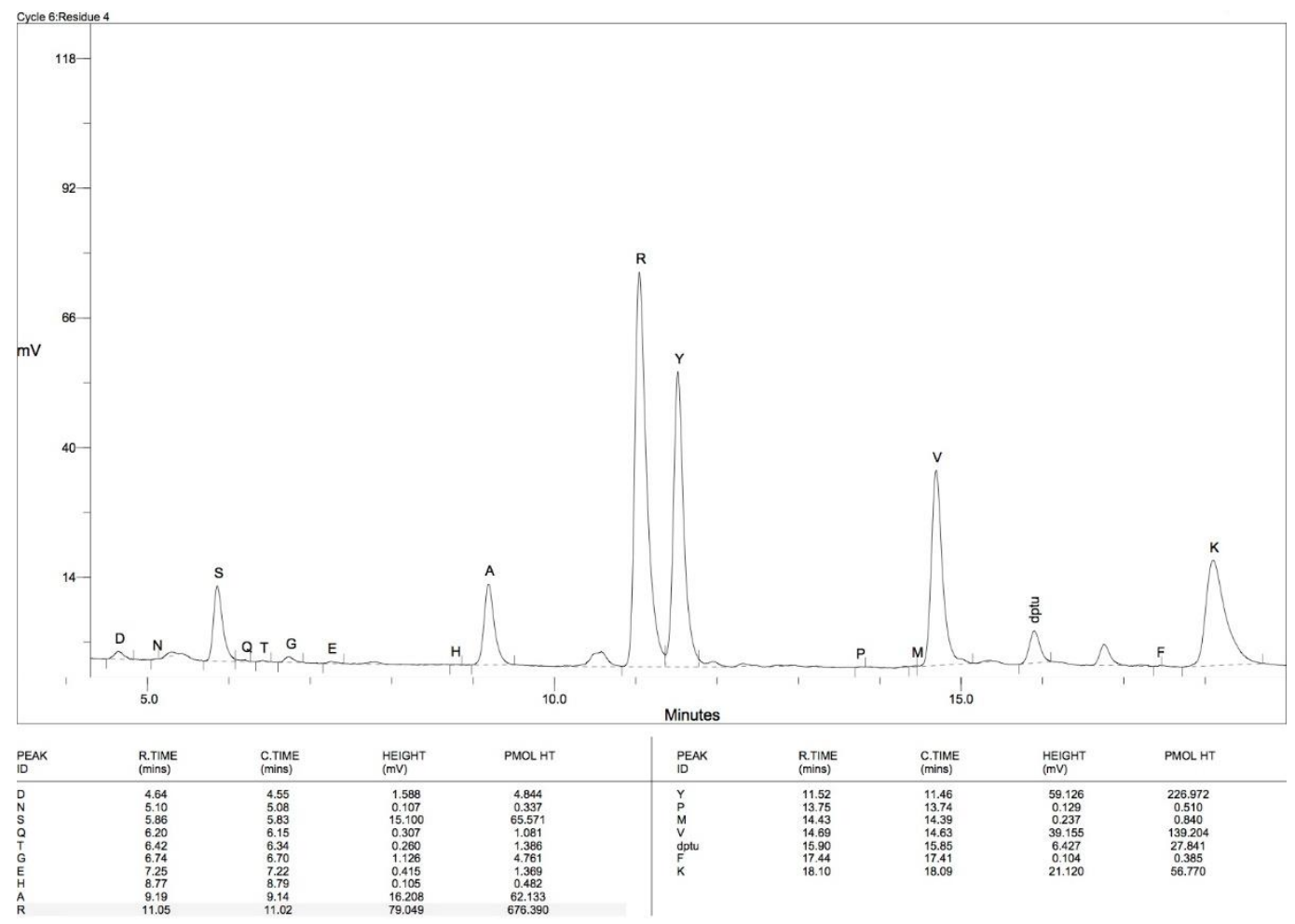

Figure S35: Edman Sequence Results for SL14 Amino Acid 4

The sequence of SL14 is R-Y-D*-R-A-S-D*-V-B-B-R-M. Arginine was present in the second randomized position.

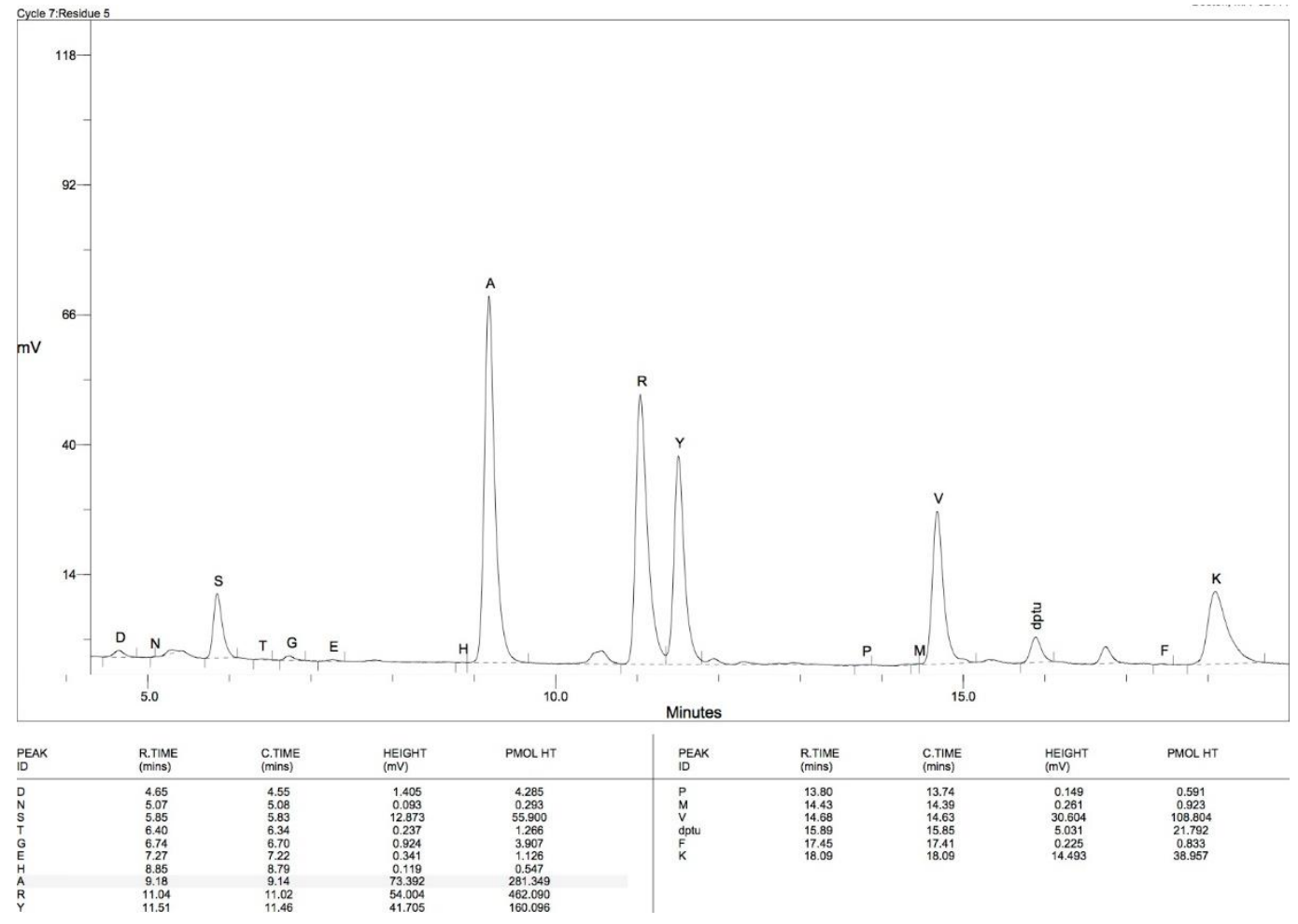

Figure S36: Edman Sequence Results for SL14 Amino Acid 5

The sequence of SL14 is R-Y-D*-R-A-S-D*-V-B-B-R-M. Alanine was present in the third randomized position. 


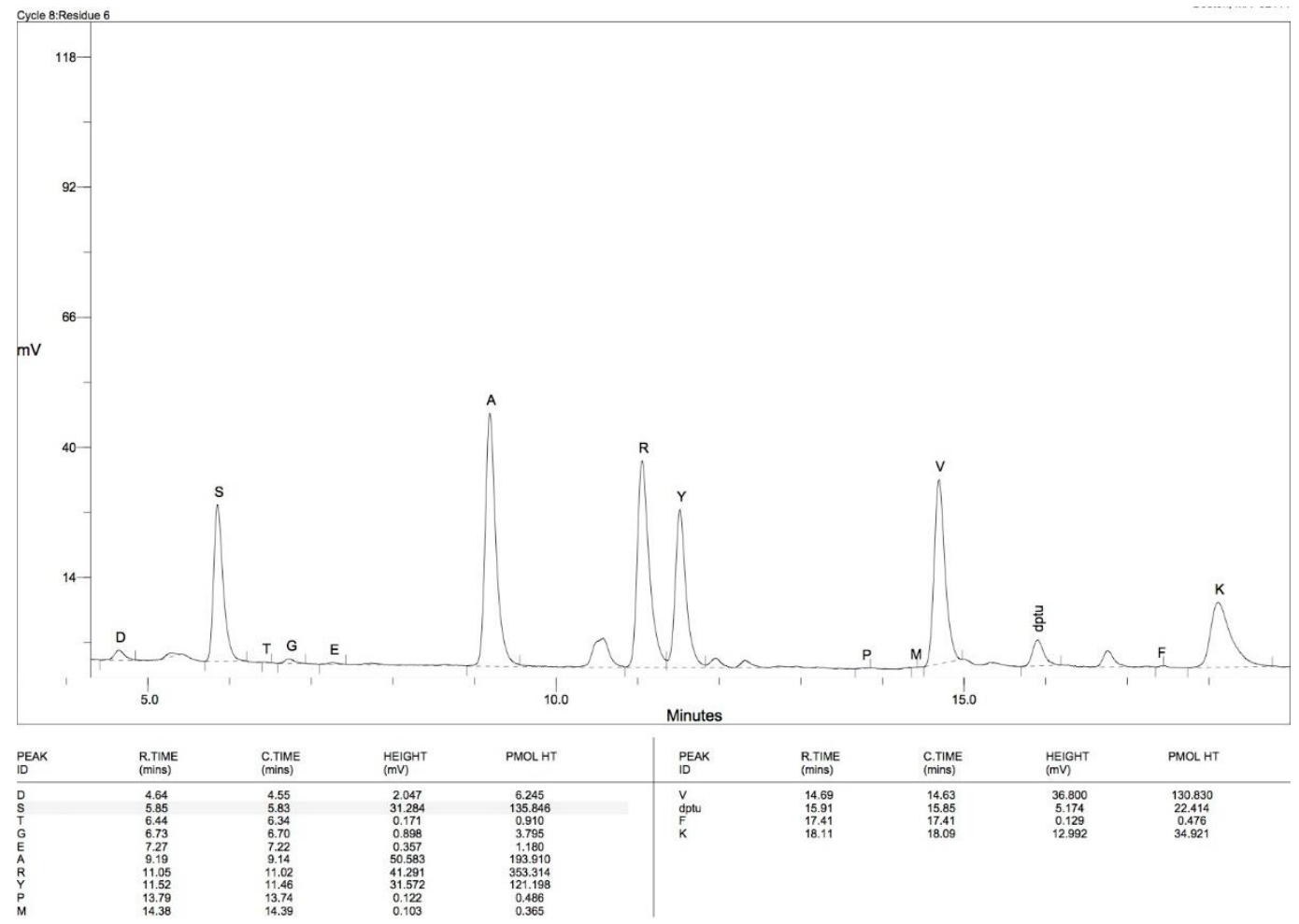

Figure S37: Edman Sequence Results for SL14 Amino Acid 6

The sequence of SL14 is R-Y-D*-R-A-S-D*-V-B-B-R-M. Serine was present in the fourth randomized position.

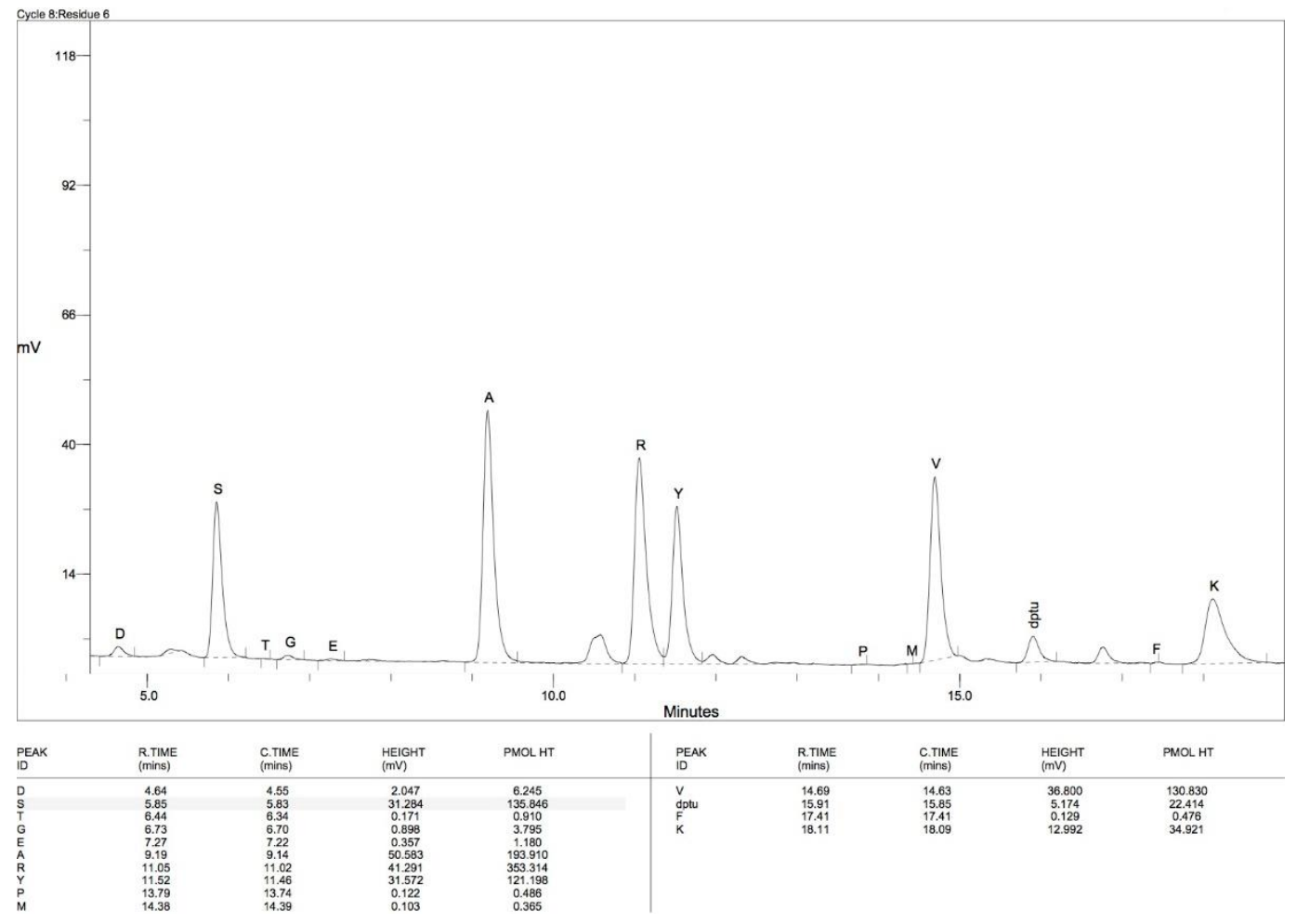

Figure S38: Edman Sequence Results for SL14 Amino Acid 7

The sequence of SL14 is R-Y-D*-R-A-S-D*-V-B-B-R-M. D* is a fixed position in the library and is not recognized by Edman Degradation. 


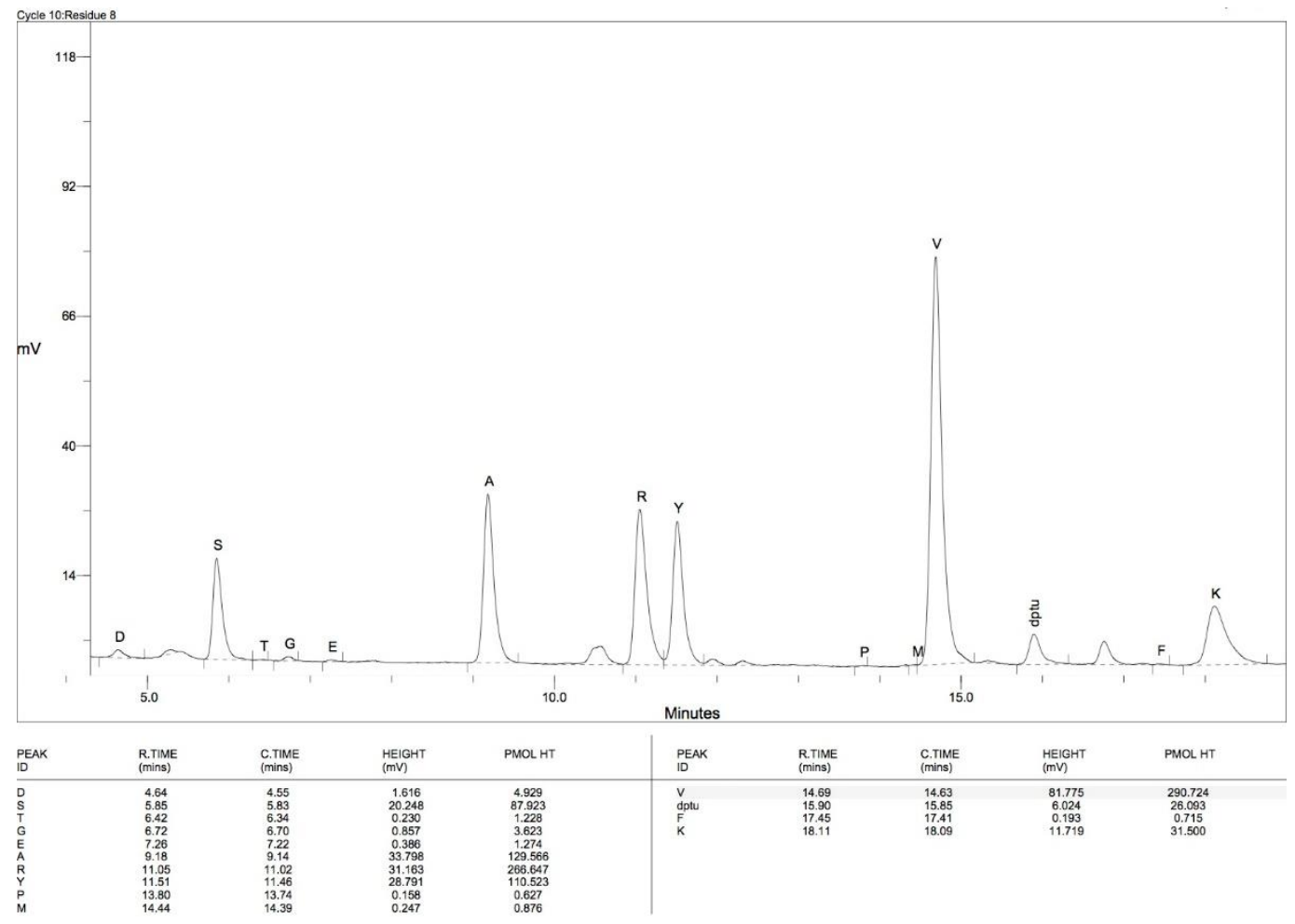

Figure S39: Edman Sequence Results for SL14 Amino Acid 8

The sequence of SL14 is R-Y-D*-R-A-S-D*-V-B-B-R-M. D* is a fixed position in the library and is not recognized by Edman Degradation. 
Validation of SL Binding: Discrimination of RWPE-1 and PC3 (SL10)

In this analysis, we used SL 10 to classify the normal prostate cell line, RWPE-1, and the highly metastatic prostate cell lines, PC3. This analysis looked at the SL hits ability to detect changes in glycosylation between cell lines of differing disease status. RWPE-1 and PC3 were chosen in this analysis because they were used in the initial single analyte screen that identified the SL hits.

\section{Group Frequencies}

$\begin{array}{cc}\text { PC3 } & \text { RWPE-1 } \\ 18 & 19\end{array}$

\section{Group means}

SL 10

\begin{tabular}{cc} 
PC3 & RWPE-1 \\
\hline 0.609 & 0.571
\end{tabular}

Between groups F-matrix -- df $=135$

\begin{tabular}{lcc} 
& PC3 & RWPE-1 \\
\cline { 2 - 3 } PC3 & 0 & \\
RWPE-1 & 1.220 & 0
\end{tabular}

\section{Wilks' lambda}

Lambda $=0.9663$

Approx. $F=1.2198$

$$
\begin{aligned}
& d f=1135 \\
& d f=135
\end{aligned}
$$

prob $=0.28$

\section{Classification functions}

\begin{tabular}{|c|c|c|c|c|c|c|}
\hline \multirow{2}{*}{$\begin{array}{l}\text { Included } \\
\text { Variable }\end{array}$} & \multirow[b]{2}{*}{ F-to-remove } & \multicolumn{5}{|c|}{ Excluded } \\
\hline & & Tolerance & 1 & Variable & F-to-enter & Tolerance \\
\hline SL 10 & 1.22 & 1.000 & | & & & \\
\hline
\end{tabular}

\begin{tabular}{lcc} 
& PC3 & RWPE-1 \\
\cline { 2 - 3 } CONSTANT & -18.344 & -16.199 \\
SL 10 & 57.912 & 54.368
\end{tabular}

Classification matrix (cases in row categories classified into columns)

\begin{tabular}{lccc} 
& PC3 & RWPE-1 & \%correct \\
\cline { 2 - 4 } PC3 & 9 & 9 & 50 \\
RWPE-1 & 7 & 12 & 63 \\
\hline Total & 16 & $\mathbf{2 1}$ & $\mathbf{5 7}$
\end{tabular}

Jackknifed classification matrix

\begin{tabular}{lccc} 
& PC3 & RWPE-1 & \%correct \\
\cline { 2 - 4 } PC3 & 9 & 9 & $\mathbf{5 0}$ \\
RWPE-1 & 7 & 12 & $\mathbf{6 3}$ \\
\hline Total & 16 & $\mathbf{2 1}$ & $\mathbf{5 7}$
\end{tabular}

Eigenvalues

0.017

Canonical correlations

0.184 
Cumulative proportion of total dispersion

$$
1.000
$$

Wilks' lambda

lambda $=0.966$

Approx.F $=1.220$

$d f=1, \quad 35$

p-tail $=0.28$

Pillai's trace

trace $=\quad 0.034$

Approx.F=

$d f=1, \quad 35$

$p$-tail $=0.28$

Lawley-Hotelling trace

trace $=\quad 0.035$

Approx.F $=1.220$

$d f=1, \quad 35$

$p$-tail $=0.28$

Canonical discriminant functions

\begin{tabular}{lc} 
& 1 \\
\cline { 2 - 2 } Constant & -5.750 \\
SL 10 & 9.755
\end{tabular}

Canonical discriminant functions -- standardized by within variances

SL $10 \quad \frac{1}{1.000}$

\section{Canonical scores of group means}

\begin{tabular}{lc} 
& 1 \\
\cline { 2 - 2 } PC3 & 0.187 \\
RWPE-1 & -0.177
\end{tabular}


For this study, we used SL 11 to classify the RWPE-1 and PC3. RWPE-1 and PC3 were chosen in this analysis because they were used in the two-colored dual analyte competitive screen that identified the SL hits.

\section{Group Frequencies}

PC3 RWPE-1

18

19

\section{Group means}

\begin{tabular}{ccc} 
& PC3 & RWPE-1 \\
\cline { 2 - 3 } SL 11 & 0.725 & 0.366
\end{tabular}

Between groups F-matrix -- df $=135$

\begin{tabular}{lcc} 
& PC3 & RWPE-1 \\
\cline { 2 - 3 } PC3 & 0 & \\
RWPE-1 & 614.471 & 0
\end{tabular}

Wilks' lambda

Lambda $=0.0539$

$d f=1135$

Approx. $F=\quad 614.4717$

$\mathrm{df}=135$

prob $=0$

Classification functions

\begin{tabular}{lcc} 
& PC3 & RWPE-1 \\
\cline { 2 - 3 } CONSTANT & -135.918 & -35.031 \\
SL 11 & 372.941 & 188.023
\end{tabular}

\begin{tabular}{|c|c|c|c|c|c|c|}
\hline \multicolumn{2}{|l|}{ Included } & \multicolumn{5}{|c|}{ Excluded } \\
\hline Variable & F-to-remove & Tolerance & 1 & Variable & F-to-enter & Tolerance \\
\hline SL 11 & 614.47 & 1.0000 & | & & & \\
\hline
\end{tabular}

Classification matrix (cases in row categories classified into columns)

\begin{tabular}{lccc} 
& PC3 & RWPE-1 & \%correct \\
\cline { 2 - 4 } PC3 & 18 & 0 & 100 \\
RWPE-1 & 0 & 19 & 100 \\
\hline Total & 18 & 19 & 100
\end{tabular}

Jackknifed classification matrix

\begin{tabular}{lccc} 
& PC3 & RWPE-1 & \%correct \\
\cline { 2 - 4 } PC3 & 18 & 0 & 100 \\
RWPE-1 & 0 & 19 & 100 \\
\hline Total & 18 & 19 & 100
\end{tabular}

Eigenvalues

17.556

Canonical correlations

0.973

Cumulative proportion of total dispersion

1.000 
Wilks' lambda

lambda =

0.054

Approx.F=

p-tail $=0$

Pillai's trace

trace $=\quad 0.946$

Approx.F $=\quad 614.471$

$d f=1, \quad 35$

p-tail $=0$

Lawley-Hotelling trace

trace $=\quad 17.556$

Approx.F= 614.471

$d f=1, \quad 35$

p-tail $=0$

Canonical discriminant functions

\begin{tabular}{lc} 
& 1 \\
\cline { 2 - 2 } Constant & -12.257 \\
SL 11 & 22.680
\end{tabular}

Canonical discriminant functions -- standardized by within variances

SL $11 \quad \frac{1}{1.000}$

Canonical scores of group means

\begin{tabular}{lc} 
& 1 \\
\cline { 2 - 2 } PC3 & 4.187 \\
RWPE-1 & -3.967
\end{tabular}


Validation of SL Binding: Discrimination of RWPE-1 and PC3 (SL12)

In this analysis, we used SL 12 to classify RWPE-1, and PC3 because they were used in the two-colored dual analyte competitive screen that identified the SL hits.

Group Frequencies

PC3 RWPE-1

18

19

\section{Group means}

\begin{tabular}{ccc} 
& PC3 & RWPE-1 \\
\cline { 2 - 3 } SL 12 & 0.739 & 0714
\end{tabular}

Between groups F-matrix -- df $=135$

\begin{tabular}{lcc} 
& PC3 & RWPE-1 \\
\cline { 2 - 3 } PC3 & 0 & \\
RWPE-1 & 0.611 & 0
\end{tabular}

Wilks' lambda

\begin{tabular}{|c|c|c|}
\hline Lambda $=$ & 0.9828 & 1135 \\
\hline Approx. F= & 0.6109 & 135 \\
\hline
\end{tabular}

prob $=0.44$

\section{Classification functions}

\begin{tabular}{lcc} 
& PC3 & RWPE-1 \\
\cline { 2 - 3 } CONSTANT & -29.716 & -27.737 \\
SL 12 & 78.425 & 75.777
\end{tabular}

\begin{tabular}{|c|c|c|c|c|c|c|}
\hline \multirow{2}{*}{$\begin{array}{l}\text { Included } \\
\text { Variable }\end{array}$} & \multirow[b]{2}{*}{ F-to-remove } & \multicolumn{5}{|c|}{ Excluded } \\
\hline & & Tolerance & I & Variable & F-to-enter & Tolerance \\
\hline SL 12 & 0.61 & 1.0000 & 1 & & & \\
\hline
\end{tabular}

Classification matrix (cases in row categories classified into columns)

\begin{tabular}{lccc} 
& PC3 & RWPE-1 & \%correct \\
\cline { 2 - 4 } PC3 & 9 & 9 & $\mathbf{5 0}$ \\
RWPE-1 & 8 & 11 & $\mathbf{5 8}$ \\
\hline Total & $\mathbf{1 7}$ & $\mathbf{2 0}$ & $\mathbf{5 4}$
\end{tabular}

Jackknifed classification matrix

\begin{tabular}{lccc} 
& PC3 & RWPE-1 & \%correct \\
\cline { 2 - 4 } PC3 & 9 & 9 & $\mathbf{5 0}$ \\
RWPE-1 & 8 & 11 & $\mathbf{5 8}$ \\
\hline Total & $\mathbf{1 7}$ & $\mathbf{2 0}$ & $\mathbf{5 4}$
\end{tabular}

Eigenvalues 0.017

Canonical correlations 0.131

Cumulative proportion of total dispersion 1.000 
Wilks' lambda

lambda =

0.983

Approx.F= 0.611

$d f=1, \quad 35$

$p$-tail $=0.44$

Pillai's trace

trace $=\quad 0.017$

Approx.F= 0.611

$d f=1, \quad 35$

$p$-tail $=0.44$

Lawley-Hotelling trace

trace $=\quad 0.017$

Approx.F= 0.611

$d f=1, \quad 35$

$p$-tail $=0.44$

Canonical discriminant functions

\begin{tabular}{lc} 
& 1 \\
\cline { 2 - 2 } Constant & -7.483 \\
SL 12 & 10.298
\end{tabular}

Canonical discriminant functions -- standardized by within variances

SL $12 \quad \frac{1}{1.000}$

Canonical scores of group means

\begin{tabular}{lc} 
& 1 \\
\cline { 2 - 2 } PC3 & 0.132 \\
RWPE-1 & -0.125
\end{tabular}


Validation of SL Binding: Discrimination of RWPE-1 and PC3 (SL13)

In this study, RWPE-1 and PC3 were classified based on the response to SL 13. This SL was identified in a single analyte screen.

\section{Group Frequencies \\ PC3 RWPE-1 \\ $18 \quad 19$}

\section{Group means}

\begin{tabular}{ccc} 
& PC3 & RWPE-1 \\
\cline { 2 - 3 } SL 13 & 0.822 & 0.790
\end{tabular}

Between groups F-matrix -- $\mathbf{d f}=135$

\begin{tabular}{lcc} 
& PC3 & RWPE-1 \\
\cline { 2 - 3 } PC3 & 0 & \\
RWPE-1 & 1.048 & 0
\end{tabular}

\section{Wilks' lambda}
Lambda $=0.9709$
$d f=1135$
Approx. $\mathrm{F}=1.0476$

prob $=0.31$

\section{Classification functions}

\begin{tabular}{lcc} 
& PC3 & RWPE-1 \\
\cline { 2 - 3 } CONSTANT & -37.558 & -34.671 \\
SL 13 & 89.628 & 86.112
\end{tabular}

\begin{tabular}{|c|c|c|c|c|c|c|}
\hline \multirow{2}{*}{$\begin{array}{l}\text { Included } \\
\text { Variable }\end{array}$} & \multirow[b]{2}{*}{ F-to-remove } & \multicolumn{5}{|c|}{ Excluded } \\
\hline & & Tolerance & | & Variable & F-to-enter & Tolerance \\
\hline SL 13 & 1.05 & 1.0000 & I & & & \\
\hline
\end{tabular}

Classification matrix (cases in row categories classified into columns)

\begin{tabular}{lccc} 
& PC3 & RWPE-1 & \%correct \\
\cline { 2 - 4 } PC3 & 9 & 9 & $\mathbf{5 0}$ \\
RWPE-1 & 8 & 11 & $\mathbf{5 8}$ \\
\hline Total & $\mathbf{1 7}$ & $\mathbf{2 0}$ & $\mathbf{5 4}$
\end{tabular}

Jackknifed classification matrix

\begin{tabular}{lccc} 
& PC3 & RWPE-1 & \%correct \\
\cline { 2 - 4 } PC3 & 9 & 9 & $\mathbf{5 0}$ \\
RWPE-1 & 9 & 10 & $\mathbf{5 3}$ \\
\hline Total & $\mathbf{1 8}$ & $\mathbf{1 9}$ & $\mathbf{5 1}$
\end{tabular}

Eigenvalues

0.030

Canonical correlations

0.170

Cumulative proportion of total dispersion 1.000 
Wilks' lambda

lambda =

0.971

Approx.F= $\quad 1.048$

$d f=1, \quad 35$

$p$-tail $=0.31$

Pillai's trace

trace $=\quad 0.029$

Approx.F $=1.048$

$d f=1, \quad 35$

$p$-tail $=0.31$

Lawley-Hotelling trace

trace $=\quad 0.030$

Approx.F $=1.048$

$d f=1, \quad 35$

$p$-tail $=0.31$

Canonical discriminant functions

\begin{tabular}{lc} 
& 1 \\
\cline { 2 - 2 } Constant & -8.411 \\
SL 13 & 10.442
\end{tabular}

Canonical discriminant functions -- standardized by within variances

SL $13 \quad \frac{1}{1.000}$

Canonical scores of group means

\begin{tabular}{lc} 
& 1 \\
\cline { 2 - 2 } PC3 & 0.173 \\
RWPE-1 & -0.164
\end{tabular}


Validation of SL Binding: Discrimination of RWPE-1 and PC3 (SL14)

In this analysis, we used SL 14 to classify RWPE-1, and PC3. SL 14 was identified from a dual dye competitive screen.

\section{Group Frequencies \\ PC3 RWPE-1 \\ $18 \quad 19$}

\section{Group means}

SL 14

\begin{tabular}{cc} 
PC3 & RWPE-1 \\
\hline 0.518 & 0.442
\end{tabular}

Between groups F-matrix -- $\mathbf{d f}=135$

\begin{tabular}{lcc} 
& PC3 & RWPE-1 \\
\cline { 2 - 3 } PC3 & 0 & \\
RWPE-1 & 70.638 & 0
\end{tabular}

Wilks' lambda

$\begin{array}{ll}\text { Lambda }= & 0.3313 \\ \text { Approx. } F= & 70.6380\end{array}$

$\mathrm{df}=1135$

$\begin{array}{ll}\mathrm{df}=135 & \text { prob }=0\end{array}$

Classification functions

\begin{tabular}{lcc} 
& PC3 & RWPE-1 \\
\cline { 2 - 3 } CONSTANT & -176.270 & -128.238 \\
SL 14 & 677.252 & 577.334
\end{tabular}

\begin{tabular}{|c|c|c|c|c|c|c|}
\hline \multirow{2}{*}{$\begin{array}{l}\text { Included } \\
\text { Variable }\end{array}$} & \multirow[b]{2}{*}{ F-to-remove } & \multicolumn{5}{|c|}{ Excluded } \\
\hline & & Tolerance & | & Variable & F-to-enter & Tolerance \\
\hline SL 14 & 70.64 & 1.0000 & I & & & \\
\hline
\end{tabular}

Classification matrix (cases in row categories classified into columns)

\begin{tabular}{lccc} 
& PC3 & RWPE-1 & \%correct \\
\cline { 2 - 4 } PC3 & 16 & 2 & 89 \\
RWPE-1 & 2 & 17 & $\mathbf{8 9}$ \\
\hline Total & 18 & 19 & 89
\end{tabular}

Jackknifed classification matrix

\begin{tabular}{lccc} 
& PC3 & RWPE-1 & \%correct \\
\cline { 2 - 4 } PC3 & 16 & 2 & 89 \\
RWPE-1 & 2 & 17 & $\mathbf{8 9}$ \\
\hline Total & 18 & 19 & $\mathbf{8 9}$
\end{tabular}

Eigenvalues

2.018

Canonical correlations

0.818

Cumulative proportion of total dispersion 1.000 
Wilks' lambda

lambda $=0.331$

Approx.F $=70.638$

$d f=1, \quad 35$

p-tail $=0$

Pillai's trace

trace $=\quad 0.669$

Approx.F $=70.638$

$d f=1, \quad 35$

$p$-tail $=0$

Lawley-Hotelling trace

trace $=\quad 2.018$

Approx. $F=\quad 70.638$

$d f=1, \quad 35$

p-tail $=0$

Canonical discriminant functions

\begin{tabular}{lc} 
& 1 \\
\cline { 2 - 2 } Constant & -17.318 \\
SL 14 & 36.144
\end{tabular}

Canonical discriminant functions -- standardized by within variances

SL $43 \quad \frac{1}{1.000}$

Canonical scores of group means

\begin{tabular}{lc} 
& 1 \\
\cline { 2 - 2 } PC3 & 1.420 \\
RWPE-1 & -1.345
\end{tabular}




\section{Validation of SL Binding: Discrimination of 6 Prostate Cell Lines}

In this analysis, we used an array composed of SL 10-14 to classify 6 prostate cell lines. This analysis looked at the SL arrays ability to detect changes in glycosylation between the different cell lines. This analysis also identified which SLs had the largest contribution on classification.

\section{Group Frequencies}

$\begin{array}{cccccc}\text { DU145 } & \text { LNCaP } & \text { PC3 } & \text { RPWE-1 } & \text { WPE1-NA22 } & \text { WPE1-NB14 } \\ 20 & 19 & 18 & 19 & 20 & 20\end{array}$

\section{Group means}

\begin{tabular}{lcccrrr} 
& DU145 & LNCaP & PC3 & RWPE-1 & WPE1-NA22 & WPE1-NB14 \\
\cline { 2 - 7 } SL 10 & 0.489 & 0.644 & 0.609 & 0.571 & 0.725 & 0.807 \\
SL 11 & 0.560 & 0.731 & 0.725 & 0.366 & 0.581 & 0.772 \\
SL 12 & 0.532 & 0.719 & 0.739 & 0.714 & 0.766 & 0.795 \\
SL 13 & 0.730 & 0.883 & 0.822 & 0.790 & 0.859 & 0.894 \\
SL 14 & 0.451 & 0.493 & 0.518 & 0.442 & 0.401 & 0.428
\end{tabular}

Between groups F-matrix -- $\mathrm{df}=5 \quad 106$

\begin{tabular}{lcccccc} 
& DU145 & LNCaP & PC3 & RWPE-1 & WPE1-NA22 & WPE1-NB14 \\
\cline { 2 - 7 } DU145 & 0.000 & & & & & \\
LNCaP & 50.411 & 0.000 & & & & \\
PC3 & 48.056 & 2.590 & 0.000 & & & \\
RWPE-1 & 42.210 & 119.280 & 112.845 & 0.000 & & \\
WPE1-NA22 & 40.959 & 37.929 & 45.975 & 52.935 & 0.000 & \\
WPE1-NB14 & 96.982 & 19.446 & 30.968 & 161.705 & 34.824 & 0.000
\end{tabular}

Wilks' lambda
Lambda $=$
0.0118
$\mathrm{df}=55110$
Approx. $F=36.3698$
$\mathrm{df}=25395$
prob $=0$

Classification functions

\begin{tabular}{lcccccc} 
& DU145 & LNCaP & PC3 & RWPE-1 & WPE1-NA22 & WPE1-NB14 \\
\cline { 2 - 7 } CONSTANT & -246.997 & -362.742 & -366.171 & -227.118 & -285.031 & -372.458 \\
SL 10 & 61.489 & 82.809 & 78.951 & 75.161 & 95.808 & 106.275 \\
SL 11 & 264.825 & 344.662 & 338.963 & 180.322 & 279.381 & 364.148 \\
SL 12 & 75.481 & 102.560 & 105.780 & 103.204 & 111.268 & 115.658 \\
SL 13 & 92.145 & 113.460 & 105.289 & 88.656 & 104.853 & 115.197 \\
SL 14 & 454.070 & 492.112 & 520.888 & 448.035 & 396.692 & 419.756
\end{tabular}

\begin{tabular}{|c|c|c|c|c|c|c|}
\hline \multicolumn{2}{|l|}{ Included } & \multicolumn{5}{|c|}{ Excluded } \\
\hline Variable & F-to-remove & Tolerance & | & Variable & F-to-enter & Tolerance \\
\hline SL 10 & 10.54 & 0.981161 & 1 & & & \\
\hline SL 11 & 125.08 & 0.967303 & I & & & \\
\hline SL 12 & 13.21 & 0.983607 & I & & & \\
\hline SL 13 & 2.75 & 0.952073 & I & & & \\
\hline SL 14 & 23.83 & 0.995105 & | & & & \\
\hline
\end{tabular}


Classification matrix (cases in row categories classified into columns)

\begin{tabular}{lccccccc} 
& DU145 & LNCaP & PC3 & RWPE-1 & WPE1-NA22 & WPE1-NB14 & \%correct \\
\cline { 2 - 8 } DU145 & 18 & 0 & 0 & 2 & 0 & 0 & $\mathbf{9 0}$ \\
LNCaP & 0 & 13 & 6 & 0 & 0 & 0 & $\mathbf{6 8}$ \\
PC3 & 0 & 5 & 13 & 0 & 0 & 0 & $\mathbf{7 2}$ \\
RWPE-1 & 0 & 0 & 0 & 18 & 1 & 0 & $\mathbf{9 5}$ \\
WPE1-NA22 & 0 & 0 & 0 & 0 & 20 & 0 & 100 \\
WPE1-NB14 & 0 & 1 & 0 & 0 & 0 & $\mathbf{9 5}$ \\
\hline Total & $\mathbf{1 8}$ & $\mathbf{1 9}$ & $\mathbf{1 9}$ & $\mathbf{2 0}$ & $\mathbf{2 1}$ & $\mathbf{1 9}$ & $\mathbf{8 7}$
\end{tabular}

Jackknifed classification matrix

\begin{tabular}{lccccccc} 
& DU145 & LNCaP & PC3 & RWPE-1 & WPE1-NA22 & WPE1-NB14 & \%correct \\
\cline { 2 - 8 } DU145 & 16 & 1 & 0 & 2 & 1 & 0 & $\mathbf{8 0}$ \\
LNCaP & 0 & 12 & 6 & 0 & 0 & 1 & 63 \\
PC3 & 0 & 7 & 11 & 0 & 0 & 0 & $\mathbf{6 1}$ \\
RWPE-1 & 0 & 0 & 0 & 18 & 1 & 0 & 95 \\
WPE1-NA22 & 0 & 0 & 0 & 0 & 20 & 0 & 19 \\
WPE1-NB14 & 0 & 1 & 0 & 0 & 0 & $\mathbf{2 0}$ & $\mathbf{9 5}$
\end{tabular}

Eigenvalues

$\begin{array}{lllll}10.339 & 2.883 & 0.838 & 0.040 & 0.005\end{array}$

Canonical correlations

$\begin{array}{lllll}0.955 & 0.862 & 0.675 & 0.196 & 0.068\end{array}$

Cumulative proportion of total dispersion
0.733
0.937
0.997
1.000
1.000

Wilks' lambda

$\begin{array}{lrl}\text { Lambda }= & 0.012 & \\ \text { Approx.F }= & 36.395 & d f=25,395\end{array}$

Pillai's trace

$\begin{array}{lrl}\text { trace }= & 2.153 & \\ \text { Approx.F } & 16.642 & d f=25,550\end{array}$

Lawley-Hotelling trace

trace $=$

14.104

Approx. $\mathrm{F}=\quad 58.899$

$d f=25,522 \quad$ p-tail $=0$

Canonical discriminant functions

\begin{tabular}{lccccc} 
& 1 & 2 & 3 & 4 & 5 \\
\cline { 2 - 5 } Constant & -19.168 & 2.476 & -14.169 & 4.002 & -3.391 \\
SL 10 & 2.840 & 6.940 & 1.782 & 1.949 & 8.510 \\
SL 11 & 19.742 & -3.644 & -5.465 & 1.169 & -2.985 \\
SL 12 & 1.926 & 5.511 & 7.983 & 3.556 & -5.868 \\
SL 13 & 3.018 & 1.344 & 0.768 & -10.517 & -1.245 \\
SL 14 & 2.626 & -21.337 & 22.261 & 0.484 & 10.977
\end{tabular}


Canonical discriminant functions -- standardized by within variances

\begin{tabular}{lccccc} 
& 1 & 2 & 3 & 4 & 5 \\
\cline { 2 - 6 } SL 10 & 0.246 & 0.602 & 0.155 & 0.169 & 0.738 \\
SL 11 & 0.954 & -0.176 & -0.264 & 0.056 & -0.144 \\
SL 12 & 0.161 & 0.462 & 0.669 & 0.298 & -0.492 \\
SL 13 & 0.278 & 0.124 & 0.071 & -0.969 & -0.115 \\
SL 14 & 0.080 & -0.651 & 0.680 & 0.015 & 0.335
\end{tabular}

Canonical scores of group means

\begin{tabular}{lccccc} 
& 1 & 2 & 3 & 4 & 5 \\
\cline { 2 - 6 } DU145 & -2.306 & -1.872 & -1.519 & 0.045 & 0.009 \\
LNCaP & 2.432 & -1.100 & 0.384 & -0.384 & 0.003 \\
PC3 & 2.142 & -1.823 & 1.028 & 0.270 & -0.048 \\
RWPE-1 & -5.408 & 0.679 & 1.000 & -0.009 & 0.056 \\
WPE1-NA22 & -0.505 & 2.203 & -0.342 & -0.026 & -0.113 \\
WPE1-NB14 & 3.710 & 1.709 & -0.379 & 0.111 & 0.090
\end{tabular}




\section{Enhanced cancer cell discrimination: Directed Partitioning and Data Reduction Methods (SL 10-14: Potential)}

To further probe the ability of the SL array we used directed partitioning to discriminate the 6 prostate cell lines based on metastatic potential. This analysis, attempted to find similarities in glycosylation between cell lines of the same disease status while finding differences between cell lines of carrying status. This analysis also identified which SLs had the largest contribution on classification.

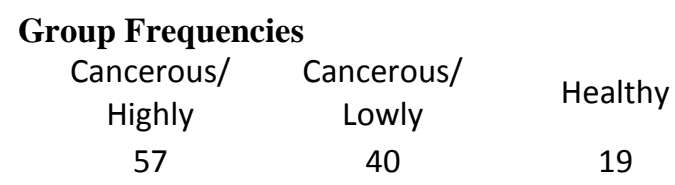

\section{Group means}

\begin{tabular}{llll} 
& Highly & Lowly & Healthy \\
\cline { 2 - 4 } SL 10 & 0.578 & 0.766 & 0.571 \\
SL 11 & 0.669 & 0.677 & 0.366 \\
SL 12 & 0.660 & 0.781 & 0.714 \\
SL 13 & 0.810 & 0.877 & 0.790 \\
SL 14 & 0.486 & 0.415 & 0.442
\end{tabular}

\begin{tabular}{lccc} 
Between groups F-matrix $-\mathbf{~ d f}=$ & $\begin{array}{c}\mathbf{5} \\
\text { Cancerous/ } \\
\text { Highly }\end{array}$ & $\begin{array}{c}\mathbf{1 0 9} \\
\text { Cancerous/ } \\
\text { Lowly }\end{array}$ & Healthy \\
\cline { 2 - 3 } $\begin{array}{l}\text { Cancerous / } \\
\text { Highly }\end{array}$ & 0.000 & & \\
$\begin{array}{l}\text { Cancerous/ } \\
\text { Lowly }\end{array}$ & 56.566 & 0.000 & \\
Healthy & 46.449 & 45.557 & 0.000
\end{tabular}

\section{Wilks' lambda}

Lambda $=0.0917 \quad \mathrm{df}=\begin{array}{lll}5 & 2 & 113\end{array}$

Approx. $F=50.1796 \quad d f=10218 \quad$ prob $=0$

\section{Classification functions}

\begin{tabular}{lccc} 
& $\begin{array}{c}\text { Cancerous/ } \\
\text { Highly }\end{array}$ & $\begin{array}{c}\text { Cancerous/ } \\
\text { Lowly }\end{array}$ & Healthy \\
\cline { 2 - 4 } CONSTANT & -100.009 & -89.729 & -93.695 \\
SL 10 & 8.124 & 31.993 & 23.266 \\
SL 11 & 4.653 & 0.790 & -46.208 \\
SL 12 & 4.529 & 20.395 & 26.438 \\
SL 13 & 46.369 & 48.753 & 44.559 \\
SL 14 & 308.955 & 225.687 & 301.602
\end{tabular}

\section{Included}

\begin{tabular}{ccc} 
Variable & F-to-remove & Tolerance \\
\hline SL 10 & 19.93 & 0.773198 \\
SL 11 & 93.35 & 0.642049 \\
SL 12 & 14.88 & 0.753423 \\
SL 13 & 0.34 & 0.846978 \\
SL 14 & 45.94 & 0.759826
\end{tabular}

\section{Excluded}

Variable F-to-enter Tolerance 
Classification matrix (cases in row categories classified into columns)

\begin{tabular}{lcccc} 
& $\begin{array}{c}\text { Cancerous/ } \\
\text { Highly }\end{array}$ & $\begin{array}{c}\text { Cancerous/ } \\
\text { Lowly }\end{array}$ & Healthy & \%correct \\
\cline { 2 - 5 } $\begin{array}{l}\text { Cancerous/ } \\
\text { Highly }\end{array}$ & 55 & 0 & 2 & 96 \\
$\begin{array}{l}\text { Cancerous/ } \\
\text { Lowly }\end{array}$ & 0 & 40 & 0 & $\mathbf{1 0 0}$ \\
Healthy & 0 & 1 & 18 & $\mathbf{9 5}$ \\
\hline Total & $\mathbf{5 5}$ & $\mathbf{4 1}$ & $\mathbf{2 0}$ & $\mathbf{9 7}$
\end{tabular}

\begin{tabular}{lcccc} 
Jackknifed classification matrix & $\begin{array}{c}\text { Cancerous/ } \\
\text { Highly }\end{array}$ & $\begin{array}{c}\text { Cancerous/ } \\
\text { Lowly }\end{array}$ & Healthy & \%correct \\
\cline { 2 - 5 } $\begin{array}{l}\text { Cancerous/ } \\
\text { Highly }\end{array}$ & 53 & 2 & 2 & 93 \\
$\begin{array}{l}\text { Cancerous/ } \\
\text { Lowly }\end{array}$ & 0 & 40 & 0 & $\mathbf{1 0 0}$ \\
Healthy & 0 & 1 & 18 & $\mathbf{9 5}$ \\
\hline Total & $\mathbf{5 3}$ & $\mathbf{4 3}$ & $\mathbf{2 0}$ & $\mathbf{9 6}$
\end{tabular}

Eigenvalues

$2.602 \quad 2.027$

Canonical correlations

$0.850 \quad 0.818$

Cumulative proportion of total dispersion

$$
0.562 \quad 1.000
$$

Wilks' lambda

Lambda $=$

Approx.F=

50.180

1.392

50.366

Approx.F=

Pillai's trace

trace $=$

Lawley-Hotelling trace

trace $=$

Approx.F=

49.989

$d f=10,216$

p-tail $=0$

\section{Canonical discriminant functions}

\begin{tabular}{lcc} 
& 1 & 2 \\
\cline { 2 - 3 } Constant & 3.444 & 1.251 \\
SL 10 & 6.844 & 0.530 \\
SL 11 & -2.370 & -12.756 \\
SL 12 & 4.858 & 3.452 \\
SL 13 & 0.597 & -0.817 \\
SL 14 & -22.691 & 10.054
\end{tabular}


Canonical discriminant functions -- standardized by within variances

\begin{tabular}{lcc} 
& 1 & 2 \\
\cline { 2 - 3 } SL 10 & 0.690 & 0.053 \\
SL 11 & -0.221 & -1.190 \\
SL 12 & 0.518 & 0.368 \\
SL 13 & 0.061 & -0.083 \\
SL 14 & -0.840 & 0.372
\end{tabular}

Canonical scores of group means

\begin{tabular}{lcc} 
& 1 & 2 \\
\cline { 2 - 3 } $\begin{array}{l}\text { Cancerous/ } \\
\text { Highly }\end{array}$ & -1.528 & -0.474 \\
$\begin{array}{l}\text { Cancerous/ } \\
\text { Lowly }\end{array}$ & 1.986 & -0.824 \\
Healthy & 0.403 & 3.155
\end{tabular}

Classification matrix (cases in row categories classified into columns)

\begin{tabular}{|c|c|c|c|c|c|c|c|}
\hline & DU145 & LNCaP & PC3 & RWPE-1 & WPE1-NA22 & WPE1-NB14 & \%correct \\
\hline DU145 & 18 & 0 & 0 & 2 & 0 & 0 & 90 \\
\hline LNCaP & 0 & 13 & 6 & 0 & 0 & 0 & 68 \\
\hline PC3 & 0 & 5 & 13 & 0 & 0 & 0 & 72 \\
\hline RWPE-1 & 0 & 0 & 0 & 18 & 1 & 0 & 95 \\
\hline WPE1-NA22 & 0 & 0 & 0 & 0 & 20 & 0 & 100 \\
\hline WPE1-NB14 & 0 & 1 & 0 & 0 & 0 & 19 & 95 \\
\hline Total & 18 & 19 & 19 & 20 & 21 & 19 & 87 \\
\hline
\end{tabular}

Jackknifed classification matrix

\begin{tabular}{lccccccc} 
& DU145 & LNCaP & PC3 & RWPE-1 & WPE1-NA22 & WPE1-NB14 & \%correct \\
\cline { 2 - 8 } DU145 & 16 & 1 & 0 & 2 & 1 & 0 & $\mathbf{8 0}$ \\
LNCaP & 0 & 12 & 6 & 0 & 0 & 1 & 63 \\
PC3 & 0 & 7 & 11 & 0 & 0 & 0 & 61 \\
RWPE-1 & 0 & 0 & 0 & 18 & 1 & 0 & $\mathbf{9 5}$ \\
WPE1-NA22 & 0 & 0 & 0 & 0 & 0 & 100 & $\mathbf{9 5}$ \\
WPE1-NB14 & 0 & 1 & 0 & 0 & 0 & $\mathbf{2 0}$ & $\mathbf{8 3}$
\end{tabular}

Eigenvalues

$\begin{array}{lllll}10.339 & 2.883 & 0.838 & 0.040 & 0.005\end{array}$

Canonical correlations

$\begin{array}{lllll}0.955 & 0.862 & 0.675 & 0.196 & 0.068\end{array}$

Cumulative proportion of total dispersion
0.733
0.937
0.997
1.000
1.000

Wilks' lambda

Lambda $=$

Approx.F=
0.012

36.395 $d f=25, \quad 395$ $p$-tail $=0$ 


\section{Pillai's trace}

$\begin{array}{lrl}\text { trace }= & 2.153 & \\ \text { Approx.F }= & 16.642 & \mathrm{df}=25,550 \quad \text { p-tail }=0\end{array}$

Lawley-Hotelling trace

trace $=\quad 14.104$

Approx.F= $\quad 58.899$

df $=25,522 \quad$ p-tail $=0$

Canonical discriminant functions

\begin{tabular}{lccccc} 
& 1 & 2 & 3 & 4 & 5 \\
\cline { 2 - 6 } Constant & -19.168 & 2.476 & -14.169 & 4.002 & -3.391 \\
SL 10 & 2.840 & 6.940 & 1.782 & 1.949 & 8.510 \\
SL 11 & 19.742 & -3.644 & -5.465 & 1.169 & -2.985 \\
SL 12 & 1.926 & 5.511 & 7.983 & 3.556 & -5.868 \\
SL 13 & 3.018 & 1.344 & 0.768 & -10.517 & -1.245 \\
SL 14 & 2.626 & -21.337 & 22.261 & 0.484 & 10.977
\end{tabular}

Canonical discriminant functions -- standardized by within variances

\begin{tabular}{lccccc} 
& 1 & 2 & 3 & 4 & 5 \\
\cline { 2 - 6 } SL 10 & 0.246 & 0.602 & 0.155 & 0.169 & 0.738 \\
SL 11 & 0.954 & -0.176 & -0.264 & 0.056 & -0.144 \\
SL 12 & 0.161 & 0.462 & 0.669 & 0.298 & -0.492 \\
SL 13 & 0.278 & 0.124 & 0.071 & -0.969 & -0.115 \\
SL 14 & 0.080 & -0.651 & 0.680 & 0.015 & 0.335
\end{tabular}

Canonical scores of group means

\begin{tabular}{lccccc} 
& 1 & 2 & 3 & 4 & 5 \\
\cline { 2 - 6 } DU145 & -2.306 & -1.872 & -1.519 & 0.045 & 0.009 \\
LNCaP & 2.432 & -1.100 & 0.384 & -0.384 & 0.003 \\
PC3 & 2.142 & -1.823 & 1.028 & 0.270 & -0.048 \\
RWPE-1 & -5.408 & 0.679 & 1.000 & -0.009 & 0.056 \\
WPE1-NA22 & -0.505 & 2.203 & -0.342 & -0.026 & -0.113 \\
WPE1-NB14 & 3.710 & 1.709 & -0.379 & 0.111 & 0.090
\end{tabular}

